\title{
Thermosensory Processing in the Drosophila Brain
}

\section{Citation}

Liu, Wendy W. 2016. Thermosensory Processing in the Drosophila Brain. Doctoral dissertation, Harvard Medical School.

\section{Permanent link}

http://nrs.harvard.edu/urn-3:HUL.InstRepos:27007730

\section{Terms of Use}

This article was downloaded from Harvard University's DASH repository, and is made available under the terms and conditions applicable to Other Posted Material, as set forth at http:// nrs.harvard.edu/urn-3:HUL.InstRepos:dash.current.terms-of-use\#LAA

\section{Share Your Story}

The Harvard community has made this article openly available.

Please share how this access benefits you. Submit a story.

Accessibility 


\section{(C) 2016 Wendy Wing-Heng Liu All rights reserved.}




\title{
Thermosensory processing in the Drosophila brain
}

\begin{abstract}
In Drosophila, just as in vertebrates, changes in external temperature are encoded by bidirectional opponent thermoreceptor cells: some cells are excited by warming and inhibited by cooling, whereas others are excited by cooling and inhibited by warming. The central circuits that process these signals are not understood. In Drosophila, a specific brain region receives input from thermoreceptor cells. Here we show that distinct genetically-identified projection neurons (PNs) in this brain region are excited by cooling, warming, or both. The PNs excited by cooling receive mainly feedforward excitation from cool thermoreceptors. In contrast, the PNs excited by warming ("warm-PNs") receive both excitation from warm thermoreceptors and crossover inhibition from cool thermoreceptors via inhibitory interneurons. Notably, this crossover inhibition elicits warming-evoked excitation, because warming suppresses tonic activity in cool thermoreceptors. This in turn disinhibits warm-PNs and sums with feedforward excitation evoked by warming. Crossover inhibition could cancel non-thermal activity (noise) that is positively-correlated among warm and cool thermoreceptor cells, while reinforcing thermal activity which is anti-correlated. Our results show how central circuits can combine signals from bidirectional opponent neurons to construct sensitive and robust neural codes.
\end{abstract}




\section{Table of Contents}

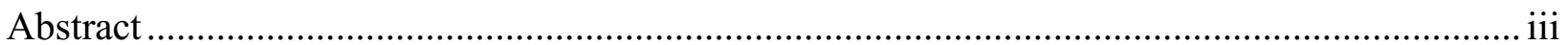

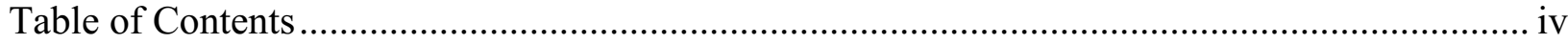

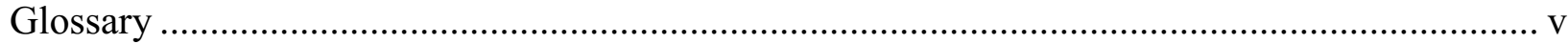

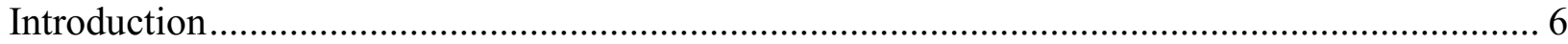

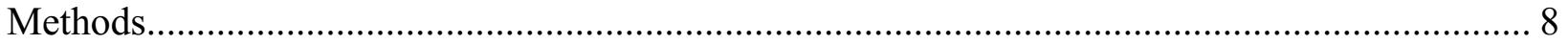

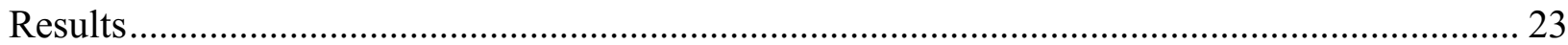

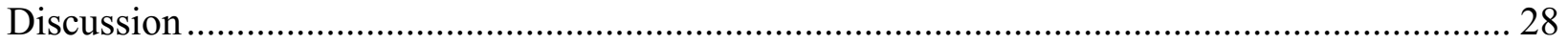

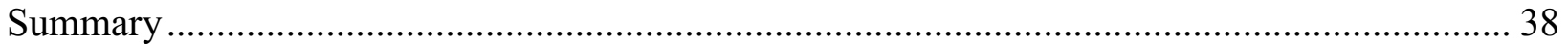

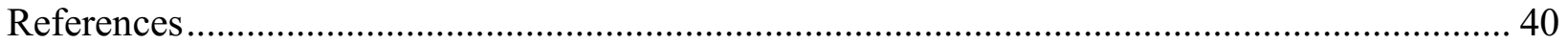

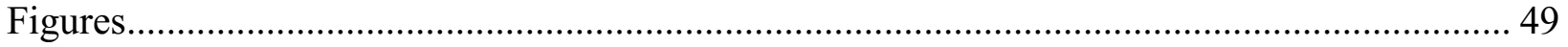


Glossary

LN - local neuron

$\mathrm{PN}$ - projection neuron 
The central circuits that process the somatic senses are poorly understood. Among the somatic senses, thermosensation is particularly intriguing from a fundamental neural coding perspective. This is because temperature is initially encoded by a population of opponent neurons which are either warm-preferring or cool-preferring, and their thermal responses are anticorrelated. Opponent channels can carry redundant signals. This raises an important question of how the central nervous system integrates information from warm and cool thermoreceptor neurons.

\section{Theories of thermosensory processing}

In the nineteenth century, Magnus Blix (1849-1904), Alfred Goldscheider (1858-1935) and Henry Herbert Donaldson (1857-1938) independently discovered that electrical stimulation of certain spots on the skin evoked thermal sensations. These were mostly cool sensations, but warmth was occasionally experienced as well. By stimulating the skin with various temperatures or electric current, the investigators were able to map out spots on the skin that produced either warm or cool sensations. A warm stimulus rarely evoked a sense of warmth from a cool spot, and vice versa. Based on these observations, they proposed that different thermal sensations arise from the stimulation of distinct receptors in the skin that give rise to only one perceptual quality (Green, 2004; Norrsell et al., 1999). This theory was later popularized by Max von Frey (1852-1932) as the specificity theory of somesthesis, which later became known as the "labeled-line" hypothesis (Ma, 2010; Prescott and Ratte, 2012). In this simplest model of thermosensory processing, warm thermoreceptor cells contribute only to the representation of warming, whereas cool cells contribute only to the representation of cooling. In support of this idea, ablating heat-activated thermoreceptor cells in the mouse abolishes behavioral responses to noxious warming but not cooling (Cavanaugh et al., 2009). Similarly, in Drosophila, silencing warm-activated thermoreceptor cells attenuates behavioral responses to warming but not cooling; conversely, silencing cool-activated thermoreceptor cells attenuates behavioral responses to cooling but not warming (Gallio et al., 2011).

An alternative model is that different thermoreceptor cells interact centrally, so that a single cell contributes to the representation of both cooling and warming (Ma, 2010; Prescott and 
Ratte, 2012). This idea could explain perceptual phenomena like the thermal grill illusion, where interlaced warm and cool objects produce the perception of burning heat. This phenomenon was first described by Thunberg in 1896. Alrutz independently discovered a similar paradoxical heat sensation by simultaneously warming adjacent warm and cool spots on the skin. The neural basis of this phenomenon was subsequently explained in work by Mackenzie, then Craig and Bushnell, among others. These studies found that two different types of primary sensory afferents are activated by cooling. Myelinated A-fibers give rise to a sensation of innocuous cool, and nonmyelinated $\mathrm{C}$-fibers causes a noxious sensation of burning heat. The nociceptive pathway is normally inhibited by the cool pathway. During application of the thermal grill, warm stimulation inhibits the cool pathway activated by A-fibers, thereby unmasking the perception of cool-induced nociception mediated by C-fibers (Craig and Bushnell, 1994; Green, 2004; Mackenzie et al., 1975). These findings argue that, contrary to the labeled-line hypothesis, there is no fixed relationship between activation of peripheral afferents and percept. Instead, coactivated pathways converge and are processed centrally to generate the ultimate percept, and the contribution of each pathway to perception depends on what other pathways are co-activated.

Electrophysiological characterization of the thermal responses of primary sensory afferents also poses problems for the labeled-lined hypothesis (Schepers and Ringkamp, 2010). Cool fibers have maximal steady state activity between 20 and $30^{\circ} \mathrm{C}$, and could account for the percept of mild cooling. However, the stimulus response function of these fibers is bell-shaped, and their activity decreases at lower temperatures when cool sensations continue to intensify. Moreover, some cool fibers can be stimulated by high temperatures in the noxious range (Campero et al., 2001; Darian-Smith et al., 1973; Dubner et al., 1975; Kenshalo and Duclaux, 1977). The discovery of two types of warm fibers similarly challenges the direct correlation between afferent activity and percept. Most warm fibers are active at temperatures over $30^{\circ} \mathrm{C}$ and most have a bell-shaped response function peaking at $40-43^{\circ} \mathrm{C}$, with minimal activity at higher noxious temperatures. However, the peak response of a subset of warm fibers is in the range of noxious heat, allowing them to signal pain in addition to warmth (Duclaux and Kenshalo, 1980; Hensel and Iggo, 1971; Hensel and Kenshalo, 1969). The stimulus-response functions of these thermal afferents suggest that warm and cool percepts cannot be explained purely by the activity of corresponding afferents. 


\section{Interactions between somatosensory modalities}

The thermal grill illusion is an example of how there may be interactions between neural circuits that encode warm, cool and pain. There is also evidence that neural signals elicited by other somatosensory modalities such as itch, and touch, also interact with thermosensory inputs (Green, 2004; Ma, 2010). These interactions can be unmasked under certain conditions. For example, mild cooling can elicit pain that is typically suppressed by touch (Green and Pope, 2003). Other examples of cross-modal interactions include suppression of pain by light touch and suppression of itch by pain (Ma, 2012). These interactions can be accentuated under pathological conditions. For patients with mechanical allodynia, light touch is perceived as painful, in part due to the unmasking of pain pathways activated by light touch that are normally under inhibition in the spinal cord (Kuner, 2010). Consistent with this idea, intrathecal injection of GABA or glycine receptor antagonists can recapitulate mechanical allodynia in animal models (Beyer et al., 1985; Roberts et al., 1986; Yaksh, 1989). In patients with chronic itch, painful stimuli promotes itch instead of suppressing it. This is again thought to be due to central disinhibition, causing painful stimuli to activate the normally inhibited itch pathways (Ma, 2010). This mechanism is supported by animal studies that showed that loss of a subset of inhibitory neurons in the spinal cord sensitizes the itch pathway and leads to similar symptoms as those found in human patients, including excessive scratching and promotion of itch by pain (Ross et al., 2010).

The common theme from these human and animal studies is that while primary sensory afferents can be highly specialized to detect specific stimuli, somatosensory percepts are generated by combining neural signals from multiple modalities rather than from a single

modality. Central inhibition that modulates interactions between multiple modalities also emerges as an important circuit element for maintaining normal perception.

\section{Spinal cord circuits and putative computations}

Neurons in the spinal dorsal horn process somatosensory information from primary sensory afferents that innervate the skin and deep tissues of the body. The afferents terminate in different laminae of the dorsal horn depending on their sensory modality. Sensory input is processed by complex circuits involving excitatory and inhibitory interneurons before being transmitted to projection neurons that relay the information to supraspinal targets, including the 
thalamus and brainstem (Todd, 2010). However, the circuits that connect primary sensory afferents to project neurons are not well understood, mainly because of the great diversity of dorsal horn neurons, which renders it difficult to comprehensively classify interneurons and projection neurons.

The superficial dorsal horn (laminae I and II) are better characterized than deep laminae. Most neurons in laminae I and II are local interneurons. A third of these are GABAergic, and a subset co-express glycine, but most are excitatory (Polgar et al., 2003; Santos et al., 2007). These neurons are heterogeneous and display a wide variety of spiking patterns and morphologies (Prescott and De Koninck, 2002; Yasaka et al., 2010). Most dorsal horn neurons receive convergent input from multiple primary afferents and from both excitatory and inhibitory interneurons. However, the specific types and relative strengths of these inputs differ between cell types (Prescott and Ratte, 2012; Todd, 2010). Laminae I projection neurons form a large proportion of the spinothalamic tract. These include nociceptive-specific neurons that respond to pinch and/or noxious heat, cool-sensitive neurons activating by cooling and inhibited by warming, warm-sensitive neurons activated by warming, as well as polymodal nociceptive cells responsive to heat, pinch and cool (Andrew and Craig, 2001b; Han et al., 1998). The receptive fields of some projection neurons can be modulated by manipulations like nerve injury, suggesting that spinal cord circuitry dynamically shapes sensitivities to different inputs (Keller et al., 2007).

Although spinal cord circuits are far from being fully understood, it is interesting to speculate on the computations that circuit motifs perform, particularly in the context of thermosensation (Prescott and Ratte, 2012). The simplest circuit motif would be a scenario where warm and cool inputs are independently processed and do not interact, as suggested by the labeled-line hypothesis (Figure 1). Alternatively, there may be interactions between warm and cool afferents. Such interactions between modalities are often studied in contexts of pathology. However, central processing enabled by these interactions could also be useful. For example, crossover inhibition arises when one pathway inhibits the opponent pathway via inhibitory interneurons (Figure 1). Such a circuit motif is common in the retina, and can shape sensitivities and receptive fields of ganglion cells (see Discussion). In a more complicated motif, inputs of warm and cool afferents can be mixed by incorporating both inhibition and excitation from the opponent pathway (Figure 1). The degree of mixing can be modulated by the balance of 
excitatory and inhibitory drive. This type of circuit motif seems to be involved in pathologic conditions like mechanical allodynia, where disinhibition unmasks pain pathways activated by touch (Kuner, 2010).

\section{The Drosophila thermosensory system}

We investigate these models of thermosensory processing in the fruit fly Drosophila. Studying sensory processing in Drosophila offers many advantages. First, there is an arsenal of genetic tools available to label and manipulate defined neuronal populations (Venken et al., 2011). Second, many Drosophila circuits are stereotyped across animals, allowing for meaningful comparisons (Berdnik et al., 2006; Hiesinger et al., 2006; Jefferis et al., 2001). Third, it has a relatively simple brain with only 100,000 neurons, which means that it may be feasible in some cases to map out all the elements of a neural circuit. The primary sensory neurons of many modalities have been identified and can be manipulated. Finally, despite its numerical simplicity, Drosophila still has a rich behavioral repertoire. Like other poikilothermic animals, Drosophila cannot regulate its core temperature internally. Instead, it relies on rapid and reliable temperature sensing to search for favorable environments, making it well-suited for studies of thermosensation. It can navigate in response to remarkably small temperature variations (Klein et al., 2015) and learn to associate thermal cues with other sensory modalities such as vision and olfaction (Ofstad et al., 2011).

Peripheral thermoreceptor cells in Drosophila reside mainly in the antenna (Sayeed and Benzer, 1996; Tang et al., 2013; Zars, 2001), and are housed inside two structures termed the arista and the sacculus (Gallio et al., 2011; Ni et al., 2013). The arista contains six cells (Foelix et al., 1989). Three cells are excited by warming and inhibited by cooling ("warm cells"), whereas the other three cells are excited by cooling and inhibited by warming ("cool cells"). The sacculus contains additional cool cells. All these cells project to a region of the brain called the proximal antennal protocerebrum (Chiang et al., 2011).

Recent studies have identified several genes involved in thermal transduction in the antenna (Gallio et al., 2011; Hamada et al., 2008; Ni et al., 2013; Tang et al., 2013). Among these, the warm receptor Gr28b. $d$ has a particularly specific expression pattern (Ni et al., 2013; Thorne and Amrein, 2008), permitting genetic access to the warm cells in the arista. Cool cells in the antenna express the TRP channel brivido in the TRPP subfamily. However, since brivido is 
expressed by many other neurons in the brain (Gallio et al., 2011), it is not thought to be the cool receptor itself. Recent unpublished work suggests that multiple ionotropic receptors may act in combination to form receptors for cooling (Ni et al., 2015).

Our work focuses on central processing downstream of peripheral thermoreceptor cells. However, it is worth noting that Drosophila senses temperature using not only peripheral thermoreceptors, but also internal sensors located in the brain. A set of warmth-sensing central neurons express the TRP channel TRPA1 and are required for certain warmth avoidance behaviors (Hamada et al., 2008). Gr28b.d and TRPA1 may play distinct roles in setting temperature preference, with $G r 28 b$. $d$ acting peripherally to control responses to rapid temperature jumps, and TRPAI acting internally to control responses to prolonged temperature rises (Ni et al., 2013). The warmth-sensing central neurons, like the peripheral thermoreceptor cells, send projections to the proximal antennal protocerebrum (Gallio et al., 2011).

\section{Summary of current study}

In this study, we investigate how the Drosophila brain processes information from warm and cool thermoreceptor cells. Prior to this work, no studies have identified neurons in the Drosophila brain that receive input from these peripheral neurons, so little was known about central thermosensory processing in fruit flies. We use in vivo whole-cell patch-clamp recordings, together with genetic and pharmacological manipulations, to dissect the functional connectivity between the peripheral thermoreceptor cells and the brain. We describe, for the first time, thermosensory PNs postsynaptic to peripheral thermoreceptor cells that are excited by cooling, warming, or both. Furthermore, our results show how signals from thermoreceptor cells are combined. As predicted by the "labeled line" model, PNs excited by cooling receive excitation from cool thermoreceptors, with little or no input from warm thermoreceptors. In contrast, the PNs excited by warming ("warm-PNs") receive excitation from warm thermoreceptors, as well as strong crossover inhibition from cool thermoreceptors via inhibitory interneurons. Warm-PNs also receive a weak excitatory input from cool thermoreceptors which is normally masked by strong crossover inhibition from the cool pathway. Our results show how opponent signals in the periphery can be combined centrally via crossover inhibition. We propose that this circuit motif may help cancel non-thermal activity in warm and cool thermoreceptor cells.

This work has been previously published (Liu et al., 2015). 


\section{Fly stocks}

The genotypes used in each figure are as follows:

Figure 4a1,2,3,4: GMR95C02-Gal4,pJFRC2-10XUAS-IVS-mCD8::GFP

Figure 4a5: Gr28b ${ }^{\mathrm{MB} 03888} ;$ GMR95C02-Gal4,pJFRC2-10XUAS-IVS-mCD8::GFP

Figure 4b1,2,3,4: GMR49B06-Gal4,pJFRC2-10XUAS-IVS-mCD8::GFP and GMR67D03Gal4,pJFRC2-10XUAS-IVS-mCD8::GFP.

Figure 4b5: Gr28b ${ }^{\mathrm{MB} 03888}$;GMR67D03-Gal4,pJFRC2-10XUAS-IVS-mCD8::GFP

Figure 8a-c: GMR95C02-Gal4,pJFRC2-10XUAS-IVS-mCD8::GFP

Figure 8d: Gr28b ${ }^{\mathrm{MB} 03888}$;GMR95C02-Gal4,pJFRC2-10XUAS-IVS-mCD8::GFP

Figure 8e-f: pLOT-HA-Ort/Gr28b.d-LexA;GMR95C02-Gal4,pJFRC2-10XUAS-IVS-

$m C D 8:: G F P /+$

Figure 8g: GMR95C02-Gal4,pJFRC2-10XUAS-IVS-mCD8::GFP (wild type) and Gr28b ${ }^{\mathrm{MB} 03888}$;GMR95C02-Gal4,pJFRC2-10XUAS-IVS-mCD8::GFP (Gr28b. $\left.\mathrm{d}^{-/}\right)$

Figure 11a-c: GMR54A03-Gal4,pJFRC2-10XUAS-IVS-mCD8::GFP

Figure 11d: Gr28b ${ }^{\mathrm{MB} 03888}$;GMR54A03-Gal4,pJFRC2-10XUAS-IVS-mCD8::GFP

Figure 11e-g: pLOT-HA-Ort/Gr28b.d-LexA;GMR54A03-Gal4,pJFRC2-10XUAS-IVS-

$m C D 8:: G F P /+$

Figure 14a-f: pJFRC7- /+;Gad1-Gal4/+

Figure 14g: 20XUAS-GCaMP3/+;Gr28b.d-Gal4/+ (warm cells) and 20XUAS-

GCaMP3/+;;GMR79C04-Gal4/+ (cool cells)

Figure 14h: GMR95C02-Gal4,pJFRC2-10XUAS-IVS-mCD8::GFP

The Gal4 lines used to drive transgene expression in specific cell types are as follows:

GMR54A03-Gal4 - 1 warm-cool-PN

GMR49B06-Gal4 - 1 slow-cool-PN

GMR67D03-Gal4 - 1 slow-cool-PN (different from that labeled by 49B06)

GMR95C02-Gal4 - 4 fast-cool-PN and 1 warm-PN

GMR52G03-Gal4 - 3 glutamatergic LNs (see Figure 15) 
GMR91H10-Gal4 - 3 glutamatergic LNs (see Figure 15)

GMR79C04-Gal4 - 3 cool thermoreceptor cells in the arista

Gr28b.d-Gal4 - 3 warm thermoreceptor cells in the arista

Gad1-Gal4 - multiple GABAergic LNs

Flies (Drosophila melanogaster) were raised on standard cornmeal agar medium supplemented with rehydrated potato flakes on a $12 \mathrm{~h} \mathrm{light/dark} \mathrm{cycle} \mathrm{at} 25^{\circ} \mathrm{C}$. All experiments were performed on adult female flies between 6 hours and 2 days post-eclosion. The GMR Gal4 lines are described in (Pfeiffer et al., 2008) and were obtained from the Bloomington Drosophila Stock Center (BDRC). The GMR line that labels the warm-cool-PN (54A03) also stochastically labeled 1-2 additional nearby somata; we were not able to obtain recordings from these additional neurons due to weak and unreliable labeling. The GMR line that labels 3 cool thermoreceptor cells in the arista (79C04) was identified based on a visual screen for expression in the proximal antennal protocerebrum and was validated using GCaMP3 imaging of the arista (Figure 14i and Figure 9); it reliably drives expression of fluorescent reporter transgenes in only three cells in the arista, in contrast to a previously-identified line, NP4486-Gal4 (Gallio et al., 2011), which can drive expression $>3$ cells in the arista on a stochastic basis (data not shown). Gr28b.d-Gal4 was first published in (Thorne and Amrein, 2008). Gadl-Gal4 (line 2B) was constructed by Toshi Kitamoto using a Gadl promoter fragment and was first published in (Mehren and Griffith, 2006), where it was called "Gad2B-Gal4". Gr28b ${ }^{\mathrm{MB} 03888}$ was reported in (Xiang et al., 2010), and also in (Ni et al., 2013) (where it was termed the "Mi" allele); we obtained it from the BDRC. pJFRC2-10XUAS-IVS-mCD8::GFP (su(Hw)attP1) and 26XLexAop2-mCD8::GFP(attP2) were reported in (Pfeiffer et al., 2010) and were obtained from the BDRC. 20XUAS-GCaMP3 (attP18) was reported in (Tian et al., 2009) and obtained from the BDRC. pJFRC7-20XUAS-IVS-mCD8::GFP (attP40) was reported in (Fisek and Wilson, 2014); prior to that report, the same transgene was described in another insertion site (Pfeiffer et al., 2010). UAS-nls:GFP was obtained from the BDRC. pLOT-HA-Ort was a generous gift from Chi-Hon Lee. 


\section{Generation of transgenic flies}

The Gr28b.d promoter fragment was PCR amplified from genomic DNA and assembled into the EcoRI/KpnI digested pBPnlsLexA::GADflUw vector (Addgene) using the Gibson Assembly Master Mix (NEB). The PCR primers used were 5'GGCCCTTTCGTCTTCAAGAATTCGTCACCATGGCCCTGCACTTCACC-3' and 5'GGGTGGCATTTTGATTGCTAGCGGTACCTCAACGTATTTTTAAAATATTTATAGTATG TA-3'. The underlined regions indicate regions of overlap with the vector. Constructs were sequenced prior to injection into $y, w ; a t t P 40$ embryos. Genetic Services, Inc. provided transgenic production services. The expression patterns of transgenic flies were verified by crossing each LexA line to a GFP reporter and examining GFP expression in the arista and the proximal antennal protocerebrum (Figure 9).

The $p L O T-H A$-Ort vector was constructed by inserting the $2 x H A$-ORT fragment from pUAST-2xHA-ORT (Takemura et al., 2011) into the pLOT vector (Lai and Lee, 2006), and injected in $y, w$ embryos via P-element transformation.

\section{Electrophysiological recordings}

In vivo whole-cell patch clamp recordings from neural somata were performed under visual control using an upright compound microscope to direct the electrode to a GFP+ soma. The fly was restrained in a platform which allowed the brain and antennae to be bathed in saline while most of the fly remained dry. Recordings were performed essentially as previously described (Wilson and Laurent, 2005), except for the following modifications detailed in (Liu and Wilson, 2013a). The head was rotated $180^{\circ}$ around the neck connective, so that the ventral side of the brain was facing upwards and therefore accessible to visualization via the waterimmersion objective above the preparation. The fly remained alive throughout the experiment even when the head was rotated in this manner, as evidenced by continual spontaneous movements of the body, as well as normal brain activity. It was necessary to rotate the head because both the PNs and the LNs that innervate the proximal antennal protocerebrum have somata ventral to the antennal lobes, and so are inaccessible when the brain is dorsal-side up.

The brain was perfused in external saline containing (in $\mathrm{mM}$ ): $103 \mathrm{NaCl}, 3 \mathrm{KCl}, 5 \mathrm{~N}$ tris(hydroxymethyl) methyl-2-aminoethane-sulfonic acid, 8 trehalose, 10 glucose, $26 \mathrm{NaHCO}_{3}, 1$ $\mathrm{NaH}_{2} \mathrm{PO}_{4}, 1.5 \mathrm{CaCl}_{2}$, and $4 \mathrm{MgCl}_{2}$ (osmolarity adjusted to $270-275 \mathrm{mOsm}$ ). The saline was 
bubbled with $95 \% \mathrm{O}_{2} / 5 \% \mathrm{CO}_{2}$ to a $\mathrm{pH}$ of 7.3 . The internal solution for patch-clamp pipettes were contained the following (in $\mathrm{mM}$ ): 140 potassium aspartate, 10 HEPES, 1 EGTA, 4 MgATP, $0.5 \mathrm{Na}_{3} \mathrm{GTP}, 1 \mathrm{KCl}$, and 13 biocytin hydrazide. The $\mathrm{pH}$ of the internal solution was adjusted to 7.2 and the osmolarity was adjusted to $\sim 265 \mathrm{mOsm}$.

In initial experiments, we filled all recorded neurons with biocytin to visualize their morphology. Some of the lines we used in this study drive Gal4 expression in more than one neuron in the region where the somata of neurons arborizing in the posterior antennal protocerebrum are located (just ventral to the antennal lobe), but we found that each morphological type of neuron had characteristic electrophysiological properties and temperature responses, and so we could accurately identify each type within the relevant Gal4 line based on these characteristics alone. Hence in subsequent recordings, neurons were classified based on these criteria and not every cell was filled. However, PN recordings made in the Gr28b.d mutant background were always filled, and LNs were also always filled.

Recordings were performed with an Axopatch 200B amplifier (Axon Instruments). Recorded voltages were low-pass filtered at $5 \mathrm{kHz}$ and digitized at $10 \mathrm{kHz}$. The seal conductance can create a small tonic depolarization in small neurons (Gouwens and Wilson, 2009) so in many experiments we injected a small amount of constant hyperpolarizing current to bring the cell back down to its native resting potential. We estimated the native resting potential of all these cell types to be $\sim 40 \mathrm{mV}$, based on measuring spontaneous spiking in cell-attached mode prior to rupturing the seal, and then matching the spontaneous spike rate in whole-cell mode (Gouwens and Wilson, 2009). Spontaneous spikes are typically not visible in cell-attached recordings from warm-PNs, and so we assumed a resting potential of $\sim 40 \mathrm{mV}$ for these cells. In most experiments where a cell was depolarized as a result of blocking synaptic inhibition, we returned the cell to its original potential before measuring its responses to thermal stimuli, so as to avoid any confounding effects of depolarization per se. All reported membrane potential values are uncorrected for the liquid junction potential, which is $-13 \mathrm{mV}$ (Gouwens and Wilson, 2009).

In some experiments, we used picrotoxin $(100 \mu \mathrm{M})$ and CGP54626 $(50 \mu \mathrm{M})$ to block synaptic inhibition mid-way through the experiment. These drugs were added to the saline perfusate. In most experiments where a cell was depolarized as a result of blocking synaptic inhibition, we returned the cell to its original potential before measuring its responses to thermal stimuli. This procedure minimized any confounding effects of depolarization per se. In warm- 
cool-PNs, blocking synaptic inhibition tended to induce oscillations in the membrane potential, and so small effects of this manipulation should be interpreted with caution.

\section{LN recordings}

Flies with Gad1-Gal4 driving pJFRC7-20XUAS-IVS-mCD8::GFP were analyzed with dual confocal immunofluorescence microscopy, using anti-CD8 and anti-GABA antibodies, as previously described (Wilson and Laurent, 2005). We found that 90\% of CD8::GFP+ cells in the cluster of cell bodies just ventral to the antennal lobe were also GABA+. Because we restricted our electrodes to cell bodies in that region, and we also filled each recorded cell with biocytin to verify that it innervated the proximal antennal protocerebrum, it is likely that almost all the LNs we recorded using this Gal4 line are GABAergic. In these experiments, around two-thirds of cells that we recorded from in the Gad1-Gal4 line did not respond to our thermal stimuli and were discarded; many of these are likely to represent antennal lobe PNs and LNs, based on the morphologies we observed when we filled a subset of these cells with biocytin. We used a similar procedure to target our electrodes to the glutamatergic LNs: flies with either GMR52G03Gal4 or GMR91H10-Gal4 driving pJFRC2-10XUAS-IVS-mCD8::GFP were immunostained using anti-CD8 and anti-dVGluT antibodies, as previously described (Liu and Wilson, 2013a). We found that all the CD8::GFP+ cells in the cluster of cell bodies just ventral to the antennal lobe were dVGluT + . As before, we restricted our electrodes to cell bodies in that region, and we filled each recorded cell with biocytin to verify that it innervated the PAP.

\section{Thermal stimulus delivery}

Because all neurons are sensitive to temperature, and because temperature gradients between the recording and ground electrodes can produce electrical artifacts, we wanted to spatially restrict temperature changes to the peripheral thermoreceptor cells in the antenna to specifically study how peripheral signals are processed in the brain. Therefore, we designed a jet micro-thermode capable of delivering a fast temperature stimulus to a $\sim 150 \mu \mathrm{m}$ target (Figure 2). The thermode system consists of two components: a room-temperature, laminar flow saline perfusion chamber (Warner Instruments, RC-26GLP), and a probe that delivers a jet of temperature-controlled saline. The fly is placed at the center of the chamber and the probe is positioned $\sim 100 \mu \mathrm{m}$ from the ipsilateral antenna, oriented so that the jet of saline flows across 
both aristae and both antennae but not the rest of the fly. The tip of the arista is immobilized with UV glue. The laminar flow of the perfusion chamber ensures that the saline jet continues on past the antenna and out of the chamber without substantial mixing with the rest of the bath. The bath temperature was kept at $23-25^{\circ} \mathrm{C}$. We verified that the baseline firing rate of all PN types adapted almost completely within a few minutes to temperature changes within this range. The hydrodynamics of the system were visualized by adding a solution of $500 \mu \mathrm{M}$ phenol red to the saline jet. We adjusted the flow rates of the perfusion chamber and saline jet until no visible mixing occurred. Simultaneous thermocouple measurements (see below) were performed to ensure that the temperature of the bath near the brain remained stable when temperature stimuli were presented to the antenna. The intrinsically thermosensitive neurons of the brain (Hamada et al., 2008) would therefore be insensitive to our stimuli. As additional controls for the spatial specificity of our thermal stimuli, we verified that cutting the antennal nerves bilaterally abolished responses in all PN types. Additionally, removing the aristae bilaterally essentially eliminated all temperature responses in the fast-cool-PNs (see Figure 4a), and it completely eliminated the responses of the warm-PNs, warm-cool-PNs, and glutamatergic LNs (data not shown).

The jet was produced by sending saline from a reservoir (pressurized to $\sim 5 \mathrm{psi}$ ) through a narrow tube that terminated in a $160 \mu \mathrm{m}$ nozzle (Small Parts, 0.0063" ID polyimide tubing, TWPT-0063-30). Upstream of the nozzle, the tube passed through a heat exchanger made from two concentric pipes. The outer pipe was fed with a constant flow of water pumped in from a hot bath $\left(\sim 40^{\circ} \mathrm{C}\right)$ or a cold bath $\left(\sim 0^{\circ} \mathrm{C}\right)$. Water from only one of the baths flowed into the heat exchanger at a time. A system of three 3-way solenoid valves selected between hot mode and cold mode.

The heat exchanger was powerful enough to bring the saline jet (as measured at the antenna) to $\sim 13^{\circ} \mathrm{C}$ in cold mode and $\sim 34^{\circ} \mathrm{C}$ in hot mode. After switching modes, the jet temperature took $\sim 7 \mathrm{~s}$ to plateau. During this slow transition, the valves could also stop flow entirely to the outer pipe (no-flow mode) when the heat exchanger was at room temperature $\left(\sim 24^{\circ} \mathrm{C}\right)$. By switching between these three modes, we could achieve slow temperature transitions between three different temperatures: $\sim 13^{\circ}, \sim 24^{\circ}$, and $\sim 34^{\circ} \mathrm{C}$. This method was the basis for our "slow cool" and "slow warm" stimuli. 
Finally, a separate "fast" solenoid valve was installed in line with the pressurized saline tube. Opening and closing this "fast" valve turned the jet on or off. When the jet is turned off, the steady laminar flow of the bath quickly washes over the antenna, bringing it back to bath temperature $\left(\sim 24^{\circ} \mathrm{C}\right)$. Switching this valve allowed for fast $(\sim 100 \mathrm{~ms})$ transitions between a hot (or cold) jet and the $\sim 24^{\circ} \mathrm{C}$ bath. This method was the basis for our "fast cool" and "fast warm" stimuli.

To produce small temperature steps, the heat exchanger was heated to $\sim 34^{\circ} \mathrm{C}$, then cooled with ice so that the jet delivered saline that was only a few degrees warmer than the bath. We then gradually cooled the heat exchanger with ice while periodically opening and closing the "fast" valve to produce a fast jump at the antenna (within $100 \mathrm{~ms}$ ) to a temperature just above $24^{\circ} \mathrm{C}$. Over several minutes, the temperature in the heat exchanger progressively fell to $24^{\circ} \mathrm{C}$, and then continued to fall. In this manner, we were able to finely sample a range of temperatures several degrees above and below $24^{\circ} \mathrm{C}$. Because here the stimulus control was performed in open loop (not closed loop, as for the steps to $13^{\circ} \mathrm{C}$ and $\sim 34^{\circ} \mathrm{C}$ ), we could not achieve precisely the same temperature stimuli in every experiment. We therefore recorded the temperature at the antenna in each stimulus trial and post hoc binned the data by temperature to construct the sensitivity plots showing average responses to small temperature steps. In the course of these experiments, we observed that central neurons did not respond when the jet temperature precisely equaled the bath temperature (to the limits of precision of our thermocouple), indicating that these neurons do not respond to any mechanical effects of the jet.

The thermal stimulus delivery device was designed and fabricated with Ofer Mazor.

\section{Temperature measurements}

Temperature measurements at the location of the antenna were made using K-type thermocouples with a 75-100 $\mu \mathrm{m}$ tip (Omega Engineering, CHAL-001 thermocouple and SMPW-CC-K-M connector). One wire of the thermocouple was threaded into a microbore Teflon tube (Cole-Parmer, EW-06417-11) to provide mechanical rigidity and insulation between the two thermocouple wires. The thermocouple was mounted on a micromanipulator and its tip was positioned entirely within the saline jet, which included $500 \mu \mathrm{M}$ phenol red for visualization. Thermocouple measurements were acquired and digitized at $99 \mathrm{~Hz}$ by a National Instruments USB-9213 Thermocouple Measurement Device. Temperature measurements displayed in the 
figures represent the mean of several experiments with the same commands to the stimulus device; because these measurements were highly consistent across experiments (generally within $1{ }^{\circ} \mathrm{C}$ of the mean), we did not measure the temperature in every experimental replicate.

\section{Histochemistry}

In some experiments (noted above), the morphology of the recorded neuron was visualized after recording by incubating the brain with a fluorescent conjugate of streptavidin, as published previously (Wilson et al., 2004). Immunohistochemistry was performed as described previously (Wilson and Laurent, 2005). Primary antibodies were obtained from the following sources (with dilutions in parentheses): mouse nc82 from the Developmental Studies Hybridoma Bank (Laissue et al., 1999) (nc82-s, 1:50), rat anti-CD8 from Invitrogen (MCD0800, 1:200), rabbit anti-GABA from Sigma(Wilson and Laurent, 2005) (A2052, 1:200), rabbit anti-dVGluT (1:500; gift of Aaron DiAntonio, Washington University, St. Louis (Daniels et al., 2008). Secondary antibodies (Invitrogen) were used at 1:250. To reconstruct neuronal morphology from biocytin fills, we hand-traced the skeletonized morphology using the Simple Neurite Tracer plugin in Fiji, using the Fill Out command to automatically generate a 3D volume, which we subsequently converted to a $z$-projection. The morphology of the slow-cool-PN has been described previously (Jenett et al., 2012).

\section{Calcium imaging}

Cool and warm cells were imaged in separate experiments. Widefield illumination was generated with a 100-W Hg arc lamp (Olympus) attenuated with a ND-25 neutral-density filter, band-pass filtered at $540-580 \mathrm{~nm}$, and delivered to the specimen through a $40 \times$ water-immersion objective mounted in an upright compound microscope (Olympus BX51WIF). Phenol red was omitted from the saline jet during these experiments. Images were acquired using a sCMOS camera (Hamamatsu ORCA-Flash4.0 V2) with a 50-ms exposure time. Thermal stimuli were delivered in "fast" mode (i.e., with a rise time of $100 \mathrm{~ms}$ or less). Data was analyzed using custom MATLAB software. Calcium imaging confirmed that Gr28b.d-Gal4 drives expression in warm thermoreceptor cells in the arista, whereas GMR79C04-Gal4 drives expression in cool thermoreceptor cells in the arista (Figure 9). 


\section{Histamine iontophoresis}

For histamine iontophoresis, a high-resistance sharp ( 100 M $\Omega)$ pulled glass micropipette was filled with a solution of $1 \mathrm{M}$ histamine dihydrochloride in saline. The pipette was inserted into the ipsilateral third antennal segment, and histamine was ejected using a $0.5-3 \mathrm{~s}$ pulse of positive current (300 nA) applied with an iontophoresis current generator (Model 260, World Precision Instruments) gated by a TTL pulse. Histamine ejection was initiated 4-5 s before the onset of the thermal stimulus. A constant negative backing current of $40 \mathrm{nA}$ was applied to retain histamine in the pipette between ejections. These ejection parameters were chosen such that there was essentially no effect of histamine iontophoresis in control genotypes that lacked the LexA transgene (Figure 10), and the brief electrical artifact produced by the iontophoresis current pulse was small. Although some thermosensory PNs arborize bilaterally, in pilot experiments where we removed the contralateral antenna we observed no clear alteration in the thermal responses of these cells, and so we performed histamine iontophoresis only in the ipsilateral antenna. We chose to iontophorese histamine into the antenna in these experiments because in pilot studies we found that iontophoresing histamine into the brain had effects on the central neurons that did not depend on the $\mathrm{pLOT-HA-Ort}$ transgene.

We also created a LexA line designed to specifically drive expression in the cool thermoreceptor cells by converting the GMR79C04-Gal4 line to a LexA line using established methods (Pfeiffer et al., 2010), and we used this to drive expression of the histamine receptor in the cool thermoreceptor cells. However, when we attempted to silence the cool cells in this manner, we did not substantially alter the thermal responses of fast-cool-PNs, which clearly receive almost all their thermal input from the arista. The failure of this positive control is likely due to low transgene expression levels in the cool thermoreceptor cells, because GFP signals driven by this LexA line were also weak.

\section{Data analysis}

Reported numbers of replicates ( $n$ values) always represent numbers of cells recorded; in almost all cases, only one cell was recorded per brain. Error bars represent s.e.m. computed across cells (experiments). No formal statistical calculations were used to pre-determine sample sizes. Sample sizes were qualitatively governed by the observed cell-to-cell reliability in the measurements we performed in pilot experiments. Our sample sizes are similar to those generally 
employed in the field. Data distribution was assumed to be normal with similar variance between the groups that were compared statistically, but this was not formally tested. All statistical tests were two-sided. In no case were flies from the same genotype assigned to distinct experimental treatments, and so no randomization procedure was needed. No blinding was performed during experiments or analysis.

Spikes were detected using custom software in Igor Pro. Peri-stimulus time histograms were generated by calculating the firing rate in 50-ms bins that overlapped by $25 \mathrm{~ms}$. Data for each cell represents an average of at least 4 trials of the same stimulus for electrophysiology experiments, and an average of 3 trials for calcium imaging experiments. All error bars represent s.e.m. computed across cells, as do the shaded bands in the peri-stimulus time histograms. For calcium imaging experiments, fluorescence was measured within a region of interest near the base of the arista that was drawn so as to contain the somata of 1-2 GCaMP-expressing cells in the same focal plane.

To compare responses to large temperature steps, we counted spikes in a 2-s period after step onset. To compare the sensitivity of cells to small temperature steps, we measured both mean temperature and mean neural activity (either firing rate or membrane potential) in a time window 100-400 ms after opening the valve for all cells except the warm-cool-PN, where we used a window of 100-200 ms after valve opening. Where we measured the mean membrane potential within a time window, we first low-pass filtered the membrane potential at $10 \mathrm{~Hz}$ to remove spikes. Because our device does not permit forward control of temperature with subdegree precision, the exact temperatures we used were slightly different in each experiment. We therefore binned the data for the sensitivity analyses according to the measured temperature in each trial, averaging together different measurements in the same temperature bin within the same experiment. For each temperature bin we then computed then mean neural activity across experiments, and also the s.e.m. across experiments to generate the sensitivity plots; the $x$-axis value of each data point in the sensitivity plots represents the middle of each temperature bin. In order to perform tests of statistical significance on the sensitivity data, we used an iterative approach to avoid a multiple comparisons error. There is good reason to think there might be a true difference for small temperature steps that would not be evident for larger temperature steps, but we did not know a priori what temperature changes these neurons might be sensitive to. Therefore, for a given pair of genotypes and a given stimulus polarity (warming or cooling), we 
first compared responses to the smallest temperature step $\left(0.125^{\circ} \mathrm{C}\right)$ using an unpaired t-test. We then adjusted the threshold for significance $(\alpha)$ to reflect the cumulative number of tests performed $(n)$ using a Bonferroni correction $(\alpha=0.05 / n)$ and proceeded to test the adjacent temperature step $\left(0.425^{\circ} \mathrm{C}\right)$, and so on. This procedure maximized our statistical power for the smallest steps. Because statistical power decreased for increasingly larger steps, this procedure is appropriate for identifying the smallest step where a difference might occur, but not for identifying the entire temperature range over which there might be a difference. 


\section{Results}

In a visual screen of $\sim 7000$ Gal4 lines (Pfeiffer et al., 2008), we identified several that label central neurons having dendrites in proximal antennal protocerebrum and axons projecting to higher brain regions. We call such cells thermosensory projection neurons (PNs). We used these Gal4 lines to drive GFP expression in small groups of PNs and performed in vivo wholecell current-clamp recordings from these neurons. We included biocytin in the patch pipette to reconstruct the morphology of individual neurons.

Because peripheral thermoreceptor cells reside in the antenna, we generated a thermal stimulus by manipulating the temperature of a stream of solution flowing over the antenna while keeping the temperature of the brain constant (Figure 2). As a control, we confirmed that cutting the axons of peripheral thermoreceptor cells abolished responses in this brain region (see Methods). We used large temperature changes to robustly activate peripheral thermoreceptor cells $\left(\sim 10^{\circ} \mathrm{C}\right)$. These large temperature changes were delivered at two speeds: fast $(\sim 100 \mathrm{~ms})$ and slow $(\sim 7 \mathrm{~s})$. We also used small steps $\left(<1^{\circ} \mathrm{C}\right.$ over $\left.\sim 100 \mathrm{~ms}\right)$ to measure sensitivity near threshold. This panel of stimuli revealed four physiological types of PNs, each with a distinctive morphology (Figure 3).

First, two PN types were excited by cooling and inhibited by warming. One type of showed strong adaptation to sustained temperature decreases (Figure $4 \mathrm{a}_{1-2}$; Figure 5). These PNs also responded better to fast cooling than to slow cooling. We term these "fast-cool-PNs". Fastcool-PNs were highly sensitive to small cooling steps (Figure $4 \mathrm{a}_{3}$ ). Fast-cool-PNs project to the lateral protocerebrum, a higher brain region that receives multimodal inputs (Ito et al., 2014).

By contrast, the second type of cool-PN showed little adaptation to large sustained temperature decreases (Figure $4 b_{1-2}$; Figure 5). Moreover, slow and fast cooling elicited similar peak firing rates (Figure $4 b_{2}$ ). We term these "slow-cool-PNs". Slow-cool-PNs project to the mushroom body, a higher brain region involved in learning and memory (Heisenberg, 2003). Slow-cool-PNs were less sensitive than fast-cool-PNs to small cooling steps (Figure $4 a_{3}, b_{3}$ ). Thus, these two cool-PN types encode different features of cool stimuli (see Discussion).

These cool-PN types have dendrites that overlap with the axons of the cool thermoreceptor cells (Figure 6). Recall that there are two structures in the antenna that contain cool cells, the arista and sacculus. Clipping the arista largely eliminated the responses of fast- 
cool-PNs (Figure 4a $\mathrm{a}_{2}$ ). By contrast, clipping the arista left the responses of slow-cool-PNs mainly intact, although it diminished the initial response to cooling, and it reduced tonic firing at room temperature (Figure $4 b_{2}$ ). All responses in slow-cool-PNs were abolished by cutting the antennal nerve (data not shown). Together, these results suggest a simple wiring scheme: fastcool-PNs receive input from the cool cells in the arista, whereas slow-cool-PNs receive input mainly from the cool cells in the sacculus, with a weaker input from cool cells in the arista.

The idea of a simple wiring scheme was reinforced by additional experiments on the cool-PNs. Namely, we observed only slight disinhibition in the cooling responses of cool-PNs when we bath-applied antagonists of inhibitory neurotransmitter receptors (Figure 7). Warming still suppressed the tonic activity of the cool-PNs when synaptic inhibition was blocked, implying that warming can inhibit these neurons by suppressing tonic excitation from cool thermoreceptor cells. Cool-PN responses were essentially unaltered by a functional null mutation in Gr28b.d, the warm receptor in the arista (Ni et al., 2013) (Figure 7). Together, these results imply that the thermal responses of the cool-PNs are driven primarily by cool thermoreceptor cells, with little role for warm thermoreceptor cells (Figure $4 a_{4}, 4 b_{4}$ ).

Unlike the representation of cooling, we found that the representation of warming is mediated by a relatively complex circuit. We identified one PN type that is excited by warming and inhibited by cooling (Figure 8a). These "warm-PNs" project to the lateral protocerebrum (Figure 3). Warm-PNs responded better to fast warming than to slow warming, and they showed adaptation to prolonged temperature increases (Figure 8b, Figure 5). Their responses were abolished by removing the arista (data not shown).

Blocking synaptic inhibition depolarized warm-PNs (by $6 \pm 1 \mathrm{mV}$, mean \pm s.e.m.), suggesting these cells are subject to tonic inhibition. Notably, blocking synaptic inhibition also unmasked weak cooling-evoked excitation in warm-PNs (Figure 8c). This implies that these PNs receive input from both warm and cool thermoreceptor cells in the arista, and weak coolingevoked excitation is normally masked by strong cooling-evoked inhibition. Moreover, although cooling hyperpolarizes these cells, it also elicits a barrage of excitatory postsynaptic potentials riding on the hyperpolarized membrane potential (Figure 8a, inset). This observation is further evidence that these cells receive excitatory input from cool thermoreceptor cells. Finally, we note that the dendrites of warm-PNs overlap with the axons of both warm and cool thermoreceptor cells (Figure 6), compatible with the idea that they receive input from both. 
Surprisingly, mutating the warm receptor $G r 28 b . d$ did not abolish excitatory responses to warming in these PNs (Figure 8d). Indeed, responses to fast warming were almost unchanged. Interestingly, however, responses to slow warming were smaller than wild type. These results suggest that there are two pathways for generating warming-evoked excitation in these PNs. Gr28b.d is necessary for generating normal responses to slow warming, but fast warming recruits a second pathway independent of Gr28b.d (see Discussion).

Based on these results, we hypothesized the cool pathway can excite these neurons in response to warming, via disinhibition. This hypothesis is motivated by the observation that cool thermoreceptor cells are tonically active and their tonic activity is suppressed by warming (Gallio et al., 2011). An interposed inhibitory neuron could relay this tonic activity in the form of tonic inhibition, and warming would withdraw this inhibition. This hypothesis predicts that blocking synaptic inhibition will eliminate the second pathway. Indeed, we found that blocking inhibition abolished warming-evoked excitation in warm-PNs in the Gr28b.d mutant (Figure 8d). This result was not observed in a wild type background: in that case, blocking inhibition potentiated warming responses rather than eliminating them (Figure 8c). As an additional control, we confirmed that blocking inhibition had essentially no effect in the cool-PNs in the Gr28b.d mutant (Figure 7).

In principle, a mutation in the warm receptor Gr28b.d might cause the circuit to re-wire, or there might be other receptor molecules that could mediate responses in warm thermoreceptor cells when Gr28b. $d$ is absent. To address these caveats, we acutely silenced the warm thermoreceptor cells themselves. We used the LexA system to selectively express a histaminegated chloride channel (Ort) in warm thermoreceptor cells under the control of the Gr28b.d promoter (Figure 9). Neurons that mis-express Ort in this manner can be acutely silenced by histamine (Liu and Wilson, 2013b). We first confirmed that simply expressing Ort in warm thermoreceptor cells did not substantially alter the responses of warm-PNs (Figure 8e). During these recordings, we then microiontophoresed histamine into the antenna to silence the warm thermoreceptor cells. PN responses to fast warming were largely intact, although responses to slow warming were significantly reduced (Figure 8f). Blocking inhibition nearly abolished warming-evoked excitation when the warm thermoreceptor cells were silenced (Figure 8f). Thus, this manipulation phenocopies the Gr28b.d mutant. These results further support a model whereby the cool pathway can cause warming-induced excitation, via disinhibition. 
Small temperature steps $\left(<1^{\circ} \mathrm{C}\right)$ yielded further insight into the two pathways which provide input to warm-PNs. Under normal conditions, warm-PNs were excited by small warming steps (Figure 8g). Blocking inhibition unmasked a response to small cooling steps (Figure 8g). Responses to small warming steps were still present in the Gr28b.d mutant, but - unlike responses to large warming steps - they were significantly reduced. Responses to small warming steps were eliminated in the mutant after inhibition was blocked (Figure 8g). These results bolster the conclusion that both the warm pathway and the cool pathway can drive excitatory responses in warm-PNs (Figure 8h). The warm pathway is most important when the rate of warming is low $\left(\sim 1^{\circ} \mathrm{C}\right.$ per s, as in our "small" stimuli and "slow large" stimuli). The cool pathway becomes dominant when the rate of warming is high $\left(\sim 10^{\circ} \mathrm{C}\right.$ over $\left.100 \mathrm{~ms}\right)$.

The fourth and final projection neuron type we identified was excited by both cooling and warming (Figure 11a). These "warm-cool-PNs" project to the lateral protocerebrum (Figure 3). They responded to fast but not slow changes in temperature, and their responses to sustained stimuli were strongly adapting (Figure 11b, Figure 5). Thus, these neurons signal rapid temperature shifts, regardless of the direction of those shifts. Removing the arista eliminated all responses in these neurons (data not shown). Blocking inhibition depolarized these cells (by $8 \pm$ $4 \mathrm{mV}$, mean \pm s.e.m.) with modest effects on their thermal responses (Figure 11c).

Notably, warming-evoked excitation persisted in warm-cool-PNs neurons in the Gr28b.d mutant (Figure 11d, Figure 12), just as we observed in warm-PNs. As before, blocking synaptic inhibition abolished warming-evoked excitation in the Gr28b.d mutant (Figure 11d). Silencing thermoreceptor warm cells with histamine again phenocopied the Gr28b.d mutation (Figure 11eg). These results imply that warming-evoked excitation in these cells arises from two sources: increased excitation from the warm pathway, and withdrawal of tonic inhibition from the cool pathway (Figure 11h). The dendrites of warm-cool-PNs overlap with the axons of both warm and cool receptor cells (Figure 6), compatible with the conclusion that they receive input from both.

These findings predict there are inhibitory neurons in this brain region that are tonically active, inhibited by warming, and excited by cooling. To test these predictions, we made recordings from inhibitory local neurons in this brain region. The main inhibitory neurotransmitters in the Drosophila brain are GABA and glutamate (Daniels et al., 2008; Enell et al., 2007; Liu and Wilson, 2013a), and so we labeled either GABAergic or glutamatergic neurons 
with GFP to target our electrodes these cells (Figure 13). In total, we recorded from 17 inhibitory neurons that arborized locally in this brain region and responded to thermal stimuli (Figure 3).

Most of the inhibitory local neurons (LNs) we recorded from (14 of 17) were tonically active at room temperature, inhibited by warming, and excited by cooling (Figure 14a-f). We term these "cool-LNs". (The three remaining LNs had the opposite tuning profile; Figure 15.) Cool-LNs were found within both the GABAergic and glutamatergic populations.

Several features of PN responses are explained by a model where cool-LNs inhibit the warm-PNs and warm-cool-PNs. First, cooling excites these LNs, which could mask coolingevoked excitation onto warm-PNs (and also attenuate cooling-evoked excitation in warm-coolPNs), except when synaptic inhibition is blocked. Conversely, warming inhibits cool-LNs, which should disinhibit PNs, allowing them to become excited even when the warm receptor is mutated or warm thermoreceptor cells are silenced, but in a manner that depends on intact synaptic inhibition. 


\section{Discussion}

In sum, our results indicate an asymmetry in the central processing of warm versus cool stimuli. Cool-PNs are driven primarily by excitation from cool thermoreceptor cells. These simple circuits evoke the classic concept of the labeled line. By contrast, warm-PNs and warmcool-PNs receive excitation from both the warm and cool pathway, as well as potent inhibition from the cool pathway. This more complex arrangement is reminiscent of some circuits in the mammalian spinal cord (see below).

\section{Crossover inhibition from the cool pathway onto warm-PNs}

Why might it be useful for warm-PNs and warm-cool-PNs to receive crossover inhibition from the cool pathway? This circuit motif may help cancel non-thermal activity (noise) that is correlated among warm and cool thermoreceptor cells. Correlated activity could arise from mechanical forces that bend the arista (Foelix et al., 1989), thereby deforming and potentially depolarizing both warm and cool cells (Sachs and Morris, 1998). Unlike the thermal responses of these cells, which are anti-correlated, correlated activity in warm and cool thermoreceptor cells would be canceled by crossover inhibition. Crossover inhibition might also increase sensitivity to small warming steps, because cool thermoreceptor cells are sensitive to warming, even though they are inhibited rather than excited by warming (Figure 14g,h). Interestingly, some insects such as the cockroach and leaf-cutting ant lack warm receptor cells entirely and yet possess both coolPNs and warm-PNs. It has been suggested that disinhibition might be the only source of warming-evoked excitation in the warm-PNs of these insects (Fischer and Tichy, 2002; Ruchty et al., 2010). In mammals, many cool peripheral thermoreceptor cells are inhibited by warming, and warm peripheral thermoreceptor cells are inhibited by cooling (Schepers and Ringkamp, 2010). Thus, the mammalian spinal cord might also use crossover inhibition, because each receptor type is informative about both warming and cooling.

Crossover inhibition is a common theme in retinal processing, where cells in the $\mathrm{ON}$ pathway inhibit those in the OFF pathway, and vice versa. In the retina, crossover inhibition has been shown to increase the sensitivity of OFF ganglion cells at low contrasts in a circuit model similar to what we describe here (Manookin et al., 2008). Responses to light decrements in OFF ganglion cells are driven not only by excitation from the OFF pathway, but also by disinhibition 
from the ON pathway mediated by amacrine cells. These amacrine cells, like the cool-LNs we describe, have tonic activity. This disinhibition plays a larger role in driving responses at low contrasts. Similarly, we also find that crossover inhibition contributes more prominently to responses of warm-PNs during small warming steps.

In the retina, crossover inhibition has other functions as well. First, it has been shown to suppress inputs from the ON pathway that spuriously appear in the OFF pathway, performing a noise cancellation function similar to the one we propose here (Renteria et al., 2006). In addition, it can correct for non-linearities in synaptic transmission (Molnar et al., 2009). A more linear postsynaptic voltage response enhances visual processing tasks such as edge detection and distinguishing luminance from contrast (Werblin, 2010). Finally, crossover inhibition in the retina has been proposed to shape the receptive fields of several types of ganglion cells and to produce temporally-precise spiking in OFF ganglion cells (Murphy and Rieke, 2006; Roska et al., 2006).

Interestingly, in the retina, the OFF pathway appears to receive more crossover inhibition than the ON pathway (Zaghloul et al., 2003). This asymmetry is analogous to what we observe in the fly thermosensory circuit, wherein the warm pathway receives more crossover inhibition from the cool pathway. In the retina, this asymmetry has been explained by the relative nonlinearities of the ON and OFF pathways. The OFF pathways receive inputs that are relatively less linear and hence require more linearization via crossover inhibition (Werblin, 2010).

\section{Excitatory input from cool peripheral cells onto warm-PNs}

It is puzzling that the warm-PNs receive excitation from the cool pathway, in addition to receiving crossover inhibition from the cool pathway. It is worth noting that many central sensory neurons receive excitation and inhibition driven by the same stimuli - i.e., co-modulated or "balanced" excitation and inhibition (Isaacson and Scanziani, 2011). In theoretical models, a balance of this type can allow neural networks to respond to stimuli more rapidly and sensitively (van Vreeswijk and Sompolinsky, 1996).

Whatever its function, this circuit motif is reminiscent of circuits in the mammalian spinal cord. In the spinal cord, some neurons in the pain pathway receive both excitation and inhibition from mechanosensory pathways, and therefore the mechanosensory inputs to these neurons are normally masked, analogous to the way that cooling-evoked excitation to the warm- 
PNs is normally masked by cooling-evoked inhibition. Some pathological states reduce inhibition from mechanosensory pathways onto these pain-pathway neurons, causing innocuous mechanical stimuli to be perceived as painful (Prescott et al., 2014; Takazawa and MacDermott, 2010). The normal function of this pathway is not well-understood. It may be that this pathway exists simply as a way to ensure that an injured part of the body is given special protection. In this view, the point of this circuit is the role it plays in pathology, because it causes the organism to shield an injury. Alternatively, this circuit may have an important function in non-pathological states, but if so, it is not clear what it might be. The relative simplicity of the Drosophila thermosensory processing circuit makes it a useful model for understanding the function of neural circuits where latent inputs are masked by co-tuned inhibition.

\section{Circuit diagrams}

In summarizing our results, we have drawn circuit diagrams with the smallest number of synapses needed to explain our data. However, some of the connections which we have schematized as direct connections may actually be indirect (polysynaptic). Our aim here is to map the functional connectivity from peripheral neurons onto central neurons, but the underlying anatomical connectivity may be more complex. In the future, a more detailed anatomical characterization of the different cell types in the proximal antennal protocerebrum will clarify this.

\section{Different PNs have differential sensitivity to the rate of temperature change}

Behavioral experiments in both Drosophila and mammals indicate that thermal perception depends partly on absolute temperature, and partly on the rate of temperature change (Kenshalo et al., 1968; Ni et al., 2013). It is therefore interesting that we found some neurons that adapt slowly to temperature changes, and others that adapt rapidly (compare e.g., the fast-coolPNs and the slow-cool-PNs, Figure 4). In other sensory systems, adaptation is often the hallmark of a highly sensitive system, because it can shift the dynamic range of a neuron to match the ambient environment (Rieke and Rudd, 2009). In this regard, it is notable that the most strongly adapting PNs are also among the most sensitive to small temperature changes (Figure 4). 


\section{Behavioral effects of thermoreceptor mutations depend on rates of temperature change}

Previous work showed that the Gr28b.d pathway is important for normal behavioral responses to temperature increases measuring $\sim 10^{\circ} \mathrm{C}$ over tens of seconds (Ni et al., 2013). This is compatible with our conclusion that the Gr28b.d pathway makes a substantial contribution to warm-PN responses when the rate of warming is $\sim 1^{\circ} \mathrm{C} / \mathrm{s}$. When warming is faster, we find that the cool pathway input to warm-PNs becomes more important. Behavioral responses to warming on even slower timescales $\left(\sim 10^{\circ} \mathrm{C}\right.$ over $\left.30 \mathrm{~min}\right)$ depend instead on a distinct thermosensory cell located inside the brain (Hamada et al., 2008; Ni et al., 2013). It would be interesting to determine how third-order thermosensory neurons integrate responses on different timescales from peripheral and internal sensors.

\section{Comparison with other descriptions of thermosensory PNs}

Our study was co-published with a study by Frank et al. which independently described distinct classes of thermosensory PNs excited by cooling, warming, or both (Frank et al., 2015). Their descriptions were generally consistent with the results of our study. They found that PNs projected to the mushroom body, lateral horn, and posterior lateral protocerebrum, which were similar to the projection patterns of the PNs described in our study. Using in vivo calcium imaging, they identified PNs that were either quickly or slowly adapting, which were consistent with our descriptions of fast-cool-PNs and slow-cool-PNs.

The studies differ in several respects. First, most of the Gal4 lines used by Frank et al. were different than those used in our study. Frank et al. selected Gal4 lines that almost exclusively labelled cells in the proximal antennal protocerebrum, with little or no labeling in other brain regions. Our selection criterion for Gal4 lines was different. We biased our selection towards lines that sparsely labelled cells near the proximal antennal protocerebrum for ease of electrophysiological recording. However, unlike Frank et al., we were able to use Gal4 lines that showed labeling both inside the PAP and outside the PAP. This is because our recording technique (targeted whole-cell patch clamp recording) is unaffected by off-target labeling, while the recording technique of Frank et al. (calcium imaging) can be more affected by off-target labeling. It is reassuring that two independent studies using different sets of Gal4 lines converge on similar descriptions of the properties of thermosensory PNs. Only one Gal4 line was used by 
both studies, and the descriptions of PNs labelled by that line were the same between the two studies.

Second, the two studies used a different stimulus design. Frank et al. delivered temperature stimuli to intact fly heads submerged in saline with pulses of $\sim 2^{\circ} \mathrm{C}$ over $1 \mathrm{~s}$. In contrast, we used two different speeds to deliver temperature changes of $\sim 10^{\circ} \mathrm{C}$ : fast $(\sim 100 \mathrm{~ms})$ and slow $(\sim 7 \mathrm{~s})$, isolated to the antennal in an intact fly. This allowed us to study how PNs respond to both fast and slow temperature changes.

Third, as noted above, Frank et al. used calcium imaging to describe the responses of PNs while we used whole-cell patch clamp recordings. Calcium imaging allows for simultaneous monitoring of the responses of multiple PN types. However, it does not provide as much information about response kinetics, membrane potential and spiking activity. In particular, it may be difficult for calcium imaging to detect inhibition. Fourth, while both studies found PNs that are excited by both warming and cooling, the description of their kinetics differs. The "warm-cool-PNs" in our study were only excited by very fast temperature changes $\left(>1^{\circ} \mathrm{C}\right.$ over $100 \mathrm{~ms})$ and the slower temperature changes we used $\left(10^{\circ} \mathrm{C}\right.$ over $\left.6 \mathrm{~s}\right)$ did not excite them. The PNs excited by both warming and cooling described by Frank et al. were excited by warming stimuli of $\sim 1^{\circ} \mathrm{C}$ over $1 \mathrm{~s}$, which was similar to our "slow" stimuli. Hence, the two studies may be describing two different cell types that were not identified by the other study. Interestingly, these were the most numerous PN type found by Frank et al. and they were shown to be required for normal temperature preference behaviors. Finally, our study focused on the functional connectivity from peripheral neurons onto central neurons and identified a prominent role for local inhibition, which was not addressed by Frank et al.

\section{Asymmetry in the number of warm versus cool PNs}

Based on sampling from the Gal4 lines we have identified, there are at least two slowcool-PNs, four fast-cool-PNs, one warm-PN, and one warm-cool-PN on each side of the Drosophila brain. This sample suggests that cool-activated neurons outnumber warm-activated neurons in this brain region. Similarly, Frank et al. found that cool-activated PNs are more numerous than warm-activated PNs (Frank et al., 2015).

Although these counts may be incomplete, they suggest an asymmetry in the number of warm versus cool PNs. Cool-activated receptor cells also outnumber warm-activated receptor 
cells in the antenna (Gallio et al., 2011). Interestingly, the same is true in the mammalian spinal cord and periphery (Andrew and Craig, 2001a; Dostrovsky and Hellon, 1978). We can speculate that this may be because the cool-activated pathway is evolutionarily more ancient, as some insects lack warm receptor cells altogether (Fischer and Tichy, 2002; Ruchty et al., 2010). Alternatively, this asymmetry may be driven by the natural statistics of thermal stimuli.

\section{Behavioral implications of circuit model}

The asymmetry in the central processing of warm versus cool makes several predictions for behavior. First, since cool-PNs receive little to no input from warm thermoreceptor cells, we predict that silencing warm thermoreceptor cells will affect the behavioral avoidance of cold. Conversely, the cool pathway contributes to warming responses in warm-PNs. Hence, we predict that silencing cool thermoreceptor cells will shift the fly's temperature preference towards warmer temperatures and reduce avoidance to heat. A previous study has examined the behavioral effects of manipulating peripheral thermosensory neurons using a two-choice temperature preference assay (Gallio et al., 2011). They found that silencing warm thermoreceptor cells attenuates behavioral responses to warming but not cooling, and silencing cool-activated thermoreceptor cells attenuates behavioral responses to cooling but not warming. This would seem to be inconsistent with our predictions. However, our results show that the contribution of the cool pathway to responses of warm-PNs is more important when the temperature changes are fast $\left(>1^{\circ} \mathrm{C}\right.$ over $\left.100 \mathrm{~ms}\right)$. The behavioral assay used by Gallio et al. 2011 did not provide much temporal resolution, and so the predicted contribution of cool peripheral neurons to warm avoidance may have been difficult to detect. Future studies using faster warming stimuli such as laser stimulation may better detect subtle changes in behavioral responses to warming when cool thermoreceptor cells are silenced.

A second prediction of our circuit model is that silencing warm thermoreceptor cells should not abolish behavioral responses to warming, especially when warming is fast. This prediction is motivated by our finding that crossover inhibition from cool thermoreceptors is sufficient to produce a near-normal level of warming-evoked excitation in the warm-PNs, even when warm peripheral neurons are silenced. Previous studies observed that silencing warm thermoreceptor cells can abolish behavioral responses to warming (Gallio et al., 2011; Ni et al., 
2013). However, again, these studies used relatively slow stimuli. Using faster stimuli, may reveal behavioral responses driven by the cool pathway.

\section{Noise in peripheral thermoreceptor cells}

We propose that crossover inhibition cancels non-thermal activity (noise) that is correlated among warm and cool thermoreceptor cells. The responses of these peripheral neurons have only been measured using calcium imaging and not with electrophysiological recordings (Gallio et al., 2011). This is, in part, due to difficulties in penetrating a recording electrode through the cuticle of the arista to gain access to these neurons. Hence, there have been no previous measurements of noise correlations in these cells. Recent unpublished worked reported a preparation for performing electrophysiological recordings from thermoreceptor neurons in the arista (Gonzalo and Garrity, 2015). Simultaneous recordings of both warm and cool thermoreceptor cells using similar techniques will elucidate the strength of noise correlations and clarify the role of crossover inhibition in sharpening tuning to thermal stimuli.

One reason why it may be beneficial for warm-PNs to integrate information from cool thermoreceptor cells is if cool thermoreceptor cells are intrinsically less noisy than warm thermoreceptor cells. To test this hypothesis, it would be informative to quantify the amount of information that warm and cool thermoreceptor cells carry about warming respectively, and to determine if warm-PNs can increase information about warming by integrating signals from warm and cool pathways.

\section{Properties of cool thermoreceptor cells in the antenna and sacculus}

We find that fast-cool-PNs receive input from the cool cells in the arista, whereas slowcool-PNs receive input mainly from the cool cells in the sacculus, with a weaker input from cool cells in the arista. The adaptation properties of these two types of cool-PNs may derive from the different response properties of peripheral cool neurons in the arista versus the sacculus. Future electrophysiological recordings from cool neurons in the arista and the sacculus can characterize the sensitivities of these cells to thermal stimuli and their adaptation properties. This may reveal how the two cool-PN types differentially transform and integrate information from parallel cool pathways in the periphery. 


\section{Expanding the range of thermal stimuli}

A major technical challenge in studying thermosensation is the difficulty of achieving precise and rapid control over thermal stimuli. In this study, we designed a novel thermal stimulus delivery device to deliver fast $\left(10^{\circ} \mathrm{C}\right.$ over $\left.\sim 100 \mathrm{~ms}\right)$, slow $\left(10^{\circ} \mathrm{C}\right.$ over $\left.\sim 7 \mathrm{~s}\right)$, and small steps stimuli $\left(<1^{\circ} \mathrm{C}\right.$ over $\left.\sim 100 \mathrm{~ms}\right)$ around room temperature. This stimulus set enabled us to show that PNs that have different adaptation kinetics and sensitivities. However, our device also has limitations. It cannot deliver temperature stimuli at any arbitrary rate, or from any arbitrary baseline temperature. A device with better control over these two variables will allow more systematic characterization of the adaptation properties of PNs.

It would also be informative to characterize typical thermal stimuli that flies encounter in a naturalistic environment, particularly their timescales. This will help determine the types of thermal stimuli that are behaviorally-relevant, and to test if PN types encode specific features of environmental stimuli.

\section{Interactions with olfaction and hygrosensation}

Many local neurons that innervate the proximal antennal protocerebrum also send projections to olfactory glomeruli (Chou et al., 2010; Liu and Wilson, 2013a). In addition, some thermosensory PNs project to the mushroom body and lateral horn, which are brain regions known for olfactory processing. These observations suggest that there is crosstalk between thermosensory and olfactory processing. Future electrophysiological and behavioral experiments using simultaneous delivery of both types of stimuli can test how these two sensory modalities interact at the level of circuits and behavior.

Relative humidity and temperature are interdependent. Because relative humidity is defined as the ratio of partial pressure of water vapor to the equilibrium vapor pressure, it will increase when temperature decreases if partial pressure of water vapor remains constant. There are hints that thermosensory and hygrosensory pathways interact in insects. At an anatomical level, cool-activated cells often reside with hygrosensitive cells in the same sensory sensillum (Tichy and Gingl, 2001). Recent unpublished work has described hygrosensitive receptor cells in the sacculus of the fly, where cool thermoreceptor cells also reside (Knecht and Garrity, 2015). At the level of receptive fields, cool thermosensory cells in the stick insect are activated by decreases in temperature as well as in the partial pressure of water vapor in ambient air (Tichy, 
2007). In our study, we chose to deliver our thermal stimuli in saline to eliminate any confounding effect of humidity. However, in a more naturalistic environment, insects often experience simultaneous changes of both temperature and humidity. It would be interesting to determine if peripheral thermoreceptor cells and thermosensory PNs also respond to changes in humidity. Using a more complicated stimulus delivery device that controls for both temperature and partial pressure of water vapor such as that described by (Tichy, 2007), the independent effects of these two variables on the responses of thermosensitive cells can be dissected.

\section{Diversity of local neurons}

The population of LNs in the proximal antennal protocerebrum that we identified is diverse. These LNs have different neurotransmitter profiles (glutamatergic or GABAergic), morphologies, and tuning properties (warm- or cool-activated). Our study focused only on characterizing inhibitory local neurons. However, there may also be excitatory local neurons that we have not identified. In fact, excitatory local neurons outnumber inhibitory ones in the spinal cord (Santos et al., 2007). Different LN types may mediate distinct functions in the thermosensory circuit. As more genetic tools become available, labeling of specific classes of neurons may be possible. Further characterization of the connectivity and response properties of LN subtypes will allow us to determine how different subtypes shape PN responses. This will help us gain insight into the function of local interneuron diversity in other circuits such as the spinal cord, where LNs are also remarkably heterogeneous (Prescott and Ratte, 2012).

\section{Higher-order brain regions in the thermosensory circuit}

Both our study and the study of Frank et al. find that thermosensory PNs project to several brain regions. These regions include the lateral protocerebrum, a region that receives multimodal inputs, and the mushroom body mushroom body, which is involved in learning and memory (Heisenberg, 2003; Ito et al., 2014). Both warm-activated and cool-activated PNs project to the lateral protocerebrum, suggesting that it is a major third-order region for thermosensory processing. In contrast, both studies found that only cool-activated PNs project to the mushroom body, a region that may mediate learned thermosensory behaviors. Previous work has shown that flies can learn to associate thermal stimuli with visual and olfactory cues (Ofstad et al., 2011). We do not know why thermosensory information is segregated into these different 
higher-order brain regions. Perhaps these regions are involved in different types of behavioral responses, or they may implement different computations. Future work is needed to characterize the responses of third-order thermosensory PNs and to assay for changes in temperature-driven behavior upon silencing PN types that project to specific brain regions. Such work will help elucidate the functions of these brain regions in thermosensory processing. 


\section{Summary}

In Drosophila, just as in vertebrates, changes in external temperature are encoded by bidirectional opponent thermoreceptor cells: some cells are excited by warming and inhibited by cooling, whereas others are excited by cooling and inhibited by warming. The central circuits that process these signals are not understood. The simplest model of thermosensory processing is that warm thermoreceptor cells contribute only to the representation of warming, whereas cool cells contribute only to the representation of cooling (the "labeled-line" hypothesis). An alternative model is that different thermoreceptor cells interact centrally, so that a single cell contributes to the representation of both cooling and warming. In this study, we investigate how the central nervous system integrates information from warm and cool thermoreceptor neurons.

For the first time, we describe neurons in the Drosophila brain that are postsynaptic to peripheral thermoreceptor cells. These thermosensory projection neurons (PNs) are excited by cooling, warming, or both. We use in vivo whole-cell patch-clamp recordings, together with genetic and pharmacological manipulations, to dissect the functional connectivity between the periphery and the brain. Our results show how signals from thermoreceptor cells are combined. As predicted by the labeled-line hypothesis, the PNs excited by cooling receive excitation from cool thermoreceptors, with little or no input from warm thermoreceptors. In contrast, the PNs excited by warming ("warm-PNs") receive excitation from warm thermoreceptors, as well as strong crossover inhibition from cool thermoreceptors via inhibitory interneurons. Warm-PNs also receive a weak excitatory input from cool thermoreceptors which is normally masked by strong crossover inhibition from the cool pathway. We show that crossover inhibition from cool thermoreceptors onto warm-PNs can give rise to warming-evoked excitation, because cool thermoreceptors are tonically active, and warming suppresses this tonic activity. This in turn disinhibits warm-PNs. Remarkably, crossover inhibition from cool thermoreceptors is sufficient to produce a near-normal level of warming-evoked excitation in the warm-PNs, even when warm peripheral neurons are silenced.

These results have interesting functional implications. First, our results show how opponent signals in the periphery can be combined centrally, by inverting the signal from one of the opponent channels and pooling it with excitation from the other channel. Crossover inhibition is a common theme in retinal processing but there are few examples of crossover inhibition 
outside the retina. Crossover inhibition in this circuit may increase central sensitivity to small temperature increases. Second, crossover inhibition may help cancel non-thermal activity (noise) that is positively-correlated among warm and cool thermoreceptor cells. Warm and cool thermoreceptor cells are likely subject to correlated mechanical and electrical noise, and crossover inhibition may be an adaptation to cancel this type of noise. Finally, the circuit we describe is reminiscent of some proposed circuits in the spinal cord where latent excitation is masked by co-tuned inhibition. We show that the weak cool excitatory input to the warm-PNs is normally masked by cool inhibitory input. Similarly, in the spinal cord, neurons in the pain pathway appear to receive excitatory input from mechanosensory neurons, which is normally masked by mechanosensory inhibitory input. Some pathological states reduce this inhibition, unmasking the mechanosensory excitation and causing innocuous mechanical stimuli to be perceived as painful. Hence, the Drosophila thermosensory processing circuit may be a useful model for understanding the function of somatosensory processing circuits where latent inputs are masked by co-tuned inhibition. 
Andrew, D., and Craig, A.D. (2001a). Spinothalamic lamina I neurones selectively responsive to cutaneous warming in cats. J Physiol 537, 489-495.

Andrew, D., and Craig, A.D. (2001b). Spinothalamic lamina I neurons selectively sensitive to histamine: a central neural pathway for itch. Nature neuroscience 4, 72-77.

Berdnik, D., Chihara, T., Couto, A., and Luo, L. (2006). Wiring stability of the adult Drosophila olfactory circuit after lesion. The Journal of neuroscience : the official journal of the Society for Neuroscience 26, 3367-3376.

Beyer, C., Roberts, L.A., and Komisaruk, B.R. (1985). Hyperalgesia induced by altered glycinergic activity at the spinal cord. Life sciences $37,875-882$.

Campero, M., Serra, J., Bostock, H., and Ochoa, J.L. (2001). Slowly conducting afferents activated by innocuous low temperature in human skin. The Journal of physiology 535, 855-865.

Cavanaugh, D.J., Lee, H., Lo, L., Shields, S.D., Zylka, M.J., Basbaum, A.I., and Anderson, D.J. (2009). Distinct subsets of unmyelinated primary sensory fibers mediate behavioral responses to noxious thermal and mechanical stimuli. Proc Natl Acad Sci U S A 106, 9075-9080.

Chiang, A.S., Lin, C.Y., Chuang, C.C., Chang, H.M., Hsieh, C.H., Yeh, C.W., Shih, C.T., Wu, J.J., Wang, G.T., Chen, Y.C., et al. (2011). Three-dimensional reconstruction of brain-wide wiring networks in Drosophila at single-cell resolution. Curr Biol 21, 1-11.

Chou, Y.H., Spletter, M.L., Yaksi, E., Leong, J.C., Wilson, R.I., and Luo, L. (2010). Diversity and wiring variability of olfactory local interneurons in the Drosophila antennal lobe. Nature neuroscience $13,439-449$.

Craig, A.D., and Bushnell, M.C. (1994). The thermal grill illusion: unmasking the burn of cold pain. Science 265, 252-255.

Daniels, R.W., Gelfand, M.V., Collins, C.A., and DiAntonio, A. (2008). Visualizing glutamatergic cell bodies and synapses in Drosophila larval and adult CNS. J Comp Neurol 508, $131-152$. 
Darian-Smith, I., Johnson, K.O., and Dykes, R. (1973). "Cold" fiber population innervating palmar and digital skin of the monkey: responses to cooling pulses. Journal of neurophysiology $36,325-346$.

Dostrovsky, J.O., and Hellon, R.F. (1978). The representation of facial temperature in the caudal trigeminal nucleus of the cat. J Physiol 277, 29-47.

Dubner, R., Sumino, R., and Wood, W.I. (1975). A peripheral "cold" fiber population responsive to innocuous and noxious thermal stimuli applied to monkey's face. Journal of neurophysiology $38,1373-1389$.

Duclaux, R., and Kenshalo, D.R., Sr. (1980). Response characteristics of cutaneous warm receptors in the monkey. Journal of neurophysiology 43, 1-15.

Enell, L., Hamasaka, Y., Kolodziejczyk, A., and Nassel, D.R. (2007). gamma-Aminobutyric acid (GABA) signaling components in Drosophila: immunocytochemical localization of GABA(B) receptors in relation to the $\mathrm{GABA}(\mathrm{A})$ receptor subunit RDL and a vesicular GABA transporter. $\mathrm{J}$ Comp Neurol 505, 18-31.

Fischer, H., and Tichy, H. (2002). Cold-receptor cells supply both cold- and warm-responsive projection neurons in the antennal lobe of the cockroach. J Comp Physiol A Neuroethol Sens Neural Behav Physiol 188, 643-648.

Fisek, M., and Wilson, R.I. (2014). Stereotyped connectivity and computations in higher-order olfactory neurons. Nat Neurosci 17, 280-288.

Foelix, R.F., Stocker, R.F., and Steinbrecht, R.A. (1989). Fine structure of a sensory organ in the arista of Drosophila melanogaster and some other dipterans. Cell and tissue research 258, 277287.

Frank, D.D., Jouandet, G.C., Kearney, P.J., Macpherson, L.J., and Gallio, M. (2015). Temperature representation in the Drosophila brain. Nature 519, 358-361.

Gallio, M., Ofstad, T.A., Macpherson, L.J., Wang, J.W., and Zuker, C.S. (2011). The coding of temperature in the Drosophila brain. Cell 144, 614-624.

Gonzalo, B., and Garrity, P.A. (2015). Electrophysiological characterization of thermoreceptor activity in Drosophila. Paper presented at: Neurobiology of Drosophila (Cold Spring Harbor). 
Gouwens, N.W., and Wilson, R.I. (2009). Signal propagation in Drosophila central neurons. J Neurosci 29, 6239-6249.

Green, B.G. (2004). Temperature perception and nociception. Journal of neurobiology 61, 13-29.

Green, B.G., and Pope, J.V. (2003). Innocuous cooling can produce nociceptive sensations that are inhibited during dynamic mechanical contact. Experimental brain research 148, 290-299.

Hamada, F.N., Rosenzweig, M., Kang, K., Pulver, S.R., Ghezzi, A., Jegla, T.J., and Garrity, P.A. (2008). An internal thermal sensor controlling temperature preference in Drosophila. Nature 454, 217-220.

Han, Z.S., Zhang, E.T., and Craig, A.D. (1998). Nociceptive and thermoreceptive lamina I neurons are anatomically distinct. Nature neuroscience 1,218-225.

Heisenberg, M. (2003). Mushroom body memoir: from maps to models. Nature reviews Neuroscience 4, 266-275.

Hensel, H., and Iggo, A. (1971). Analysis of cutaneous warm and cold fibres in primates. Pflugers Archiv : European journal of physiology 329, 1-8.

Hensel, H., and Kenshalo, D.R. (1969). Warm receptors in the nasal region of cats. The Journal of physiology 204, 99-112.

Hiesinger, P.R., Zhai, R.G., Zhou, Y., Koh, T.W., Mehta, S.Q., Schulze, K.L., Cao, Y., Verstreken, P., Clandinin, T.R., Fischbach, K.F., et al. (2006). Activity-independent prespecification of synaptic partners in the visual map of Drosophila. Current biology : CB 16, 1835-1843.

Isaacson, J.S., and Scanziani, M. (2011). How inhibition shapes cortical activity. Neuron 72, 231-243.

Ito, K., Shinomiya, K., Ito, M., Armstrong, J.D., Boyan, G., Hartenstein, V., Harzsch, S., Heisenberg, M., Homberg, U., Jenett, A., et al. (2014). A systematic nomenclature for the insect brain. Neuron 81, 755-765.

Jefferis, G.S., Marin, E.C., Stocker, R.F., and Luo, L. (2001). Target neuron prespecification in the olfactory map of Drosophila. Nature 414, 204-208. 
Jenett, A., Rubin, G.M., Ngo, T.T., Shepherd, D., Murphy, C., Dionne, H., Pfeiffer, B.D., Cavallaro, A., Hall, D., Jeter, J., et al. (2012). A GAL4-driver line resource for Drosophila neurobiology. Cell Rep 2, 991-1001.

Keller, A.F., Beggs, S., Salter, M.W., and De Koninck, Y. (2007). Transformation of the output of spinal lamina I neurons after nerve injury and microglia stimulation underlying neuropathic pain. Molecular pain 3, 27.

Kenshalo, D.R., and Duclaux, R. (1977). Response characteristics of cutaneous cold receptors in the monkey. Journal of neurophysiology 40, 319-332.

Kenshalo, D.R., Holmes, C.E., and Wood, P.B. (1968). Warm and cool thresholds as a function of the rate of stimulus temperature change. Percept Psychophys 3, 81-84.

Klein, M., Afonso, B., Vonner, A.J., Hernandez-Nunez, L., Berck, M., Tabone, C.J., Kane, E.A., Pieribone, V.A., Nitabach, M.N., Cardona, A., et al. (2015). Sensory determinants of behavioral dynamics in Drosophila thermotaxis. Proceedings of the National Academy of Sciences of the United States of America 112, E220-229.

Knecht, Z.A., and Garrity, P.A. (2015). The molecular and cellular basis of insect hygrosensation. Paper presented at: Neurobiology of Drosophila (Cold Spring Harbor).

Kuner, R. (2010). Central mechanisms of pathological pain. Nature medicine 16, 1258-1266.

Lai, S.L., and Lee, T. (2006). Genetic mosaic with dual binary transcriptional systems in Drosophila. Nat Neurosci 9, 703-709.

Laissue, P.P., Reiter, C., Hiesinger, P.R., Halter, S., Fischbach, K.F., and Stocker, R.F. (1999). Three-dimensional reconstruction of the antennal lobe in Drosophila melanogaster. J Comp Neurol 405, 543-552.

Liu, W.W., Mazor, O., and Wilson, R.I. (2015). Thermosensory processing in the Drosophila brain. Nature 519, 353-357.

Liu, W.W., and Wilson, R.I. (2013a). Glutamate is an inhibitory neurotransmitter in the Drosophila olfactory system. Proceedings of the National Academy of Sciences of the United States of America 110, 10294-10299. 
Liu, W.W., and Wilson, R.I. (2013b). Transient and specific inactivation of Drosophila neurons in vivo using a native ligand-gated ion channel. Curr Biol 23, 1202-1208.

Ma, Q. (2010). Labeled lines meet and talk: population coding of somatic sensations. The Journal of clinical investigation 120,3773-3778.

Ma, Q. (2012). Population coding of somatic sensations. Neuroscience bulletin 28, 91-99.

Mackenzie, R.A., Burke, D., Skuse, N.F., and Lethlean, A.K. (1975). Fibre function and perception during cutaneous nerve block. Journal of neurology, neurosurgery, and psychiatry 38 , 865-873.

Manookin, M.B., Beaudoin, D.L., Ernst, Z.R., Flagel, L.J., and Demb, J.B. (2008). Disinhibition combines with excitation to extend the operating range of the OFF visual pathway in daylight. The Journal of neuroscience : the official journal of the Society for Neuroscience 28, 4136-4150.

Mehren, J.E., and Griffith, L.C. (2006). Cholinergic neurons mediate CaMKII-dependent enhancement of courtship suppression. Learn Mem 13, 686-689.

Molnar, A., Hsueh, H.A., Roska, B., and Werblin, F.S. (2009). Crossover inhibition in the retina: circuitry that compensates for nonlinear rectifying synaptic transmission. Journal of computational neuroscience $27,569-590$.

Murphy, G.J., and Rieke, F. (2006). Network variability limits stimulus-evoked spike timing precision in retinal ganglion cells. Neuron 52, 511-524.

Ni, L., Bronk, P., Chang, E.C., Lowell, A.M., Flam, J.O., Panzano, V.C., Theobald, D.L., Griffith, L.C., and Garrity, P.A. (2013). A gustatory receptor paralogue controls rapid warmth avoidance in Drosophila. Nature 500, 580-584.

Ni, L., Klein, M., Knecht, Z., Gonzalo, B., Silbering, A., Bell, R., Benton, R., Samuel, A., and Garrity, P.A. (2015). Ionotropic receptors mediate cool sensing in Drosophila. Paper presented at: Neurobiology of Drosophila (Cold Spring Harbor).

Norrsell, U., Finger, S., and Lajonchere, C. (1999). Cutaneous sensory spots and the "law of specific nerve energies": history and development of ideas. Brain research bulletin 48, 457-465. 
Ofstad, T.A., Zuker, C.S., and Reiser, M.B. (2011). Visual place learning in Drosophila melanogaster. Nature 474, 204-207.

Pfeiffer, B., Ngo, T.-T.B., Hibbard, K.L., Murphy, C., Jenett, A., Truman, J.W., and Rubin, G.M. (2010). Refinement of tools for targeted gene expression in Drosophila. Genetics 186, 735-755.

Pfeiffer, B.D., Jenett, A., Hammonds, A.S., Ngo, T.T., Misra, S., Murphy, C., Scully, A., Carlson, J.W., Wan, K.H., Laverty, T.R., et al. (2008). Tools for neuroanatomy and neurogenetics in Drosophila. Proc Natl Acad Sci U S A 105, 9715-9720.

Polgar, E., Hughes, D.I., Riddell, J.S., Maxwell, D.J., Puskar, Z., and Todd, A.J. (2003). Selective loss of spinal GABAergic or glycinergic neurons is not necessary for development of thermal hyperalgesia in the chronic constriction injury model of neuropathic pain. Pain 104, 229239.

Prescott, S.A., and De Koninck, Y. (2002). Four cell types with distinctive membrane properties and morphologies in lamina I of the spinal dorsal horn of the adult rat. The Journal of physiology $539,817-836$.

Prescott, S.A., Ma, Q., and De Koninck, Y. (2014). Normal and abnormal coding of somatosensory stimuli causing pain. Nat Neurosci 17, 183-191.

Prescott, S.A., and Ratte, S. (2012). Pain processing by spinal microcircuits: afferent combinatorics. Current opinion in neurobiology 22, 631-639.

Renteria, R.C., Tian, N., Cang, J., Nakanishi, S., Stryker, M.P., and Copenhagen, D.R. (2006). Intrinsic ON responses of the retinal OFF pathway are suppressed by the ON pathway. The Journal of neuroscience : the official journal of the Society for Neuroscience 26, 11857-11869.

Rieke, F., and Rudd, M.E. (2009). The challenges natural images pose for visual adaptation. Neuron 64, 605-616.

Roberts, L.A., Beyer, C., and Komisaruk, B.R. (1986). Nociceptive responses to altered GABAergic activity at the spinal cord. Life sciences 39, 1667-1674.

Roska, B., Molnar, A., and Werblin, F.S. (2006). Parallel processing in retinal ganglion cells: how integration of space-time patterns of excitation and inhibition form the spiking output. Journal of neurophysiology 95, 3810-3822. 
Ross, S.E., Mardinly, A.R., McCord, A.E., Zurawski, J., Cohen, S., Jung, C., Hu, L., Mok, S.I., Shah, A., Savner, E.M., et al. (2010). Loss of inhibitory interneurons in the dorsal spinal cord and elevated itch in Bhlhb5 mutant mice. Neuron 65, 886-898.

Ruchty, M., Helmchen, F., Wehner, R., and Kleineidam, C.J. (2010). Representation of thermal information in the antennal lobe of leaf-cutting ants. Front Behav Neurosci 4, 174.

Sachs, F., and Morris, C.E. (1998). Mechanosensitive Ion Channels in Non-Specialized Cells. In Rev Physiol Biochem Pharmacol (Berlin Heidelberg: Springer), pp. 1-77.

Santos, S.F., Rebelo, S., Derkach, V.A., and Safronov, B.V. (2007). Excitatory interneurons dominate sensory processing in the spinal substantia gelatinosa of rat. The Journal of physiology $581,241-254$.

Sayeed, O., and Benzer, S. (1996). Behavioral genetics of thermosensation and hygrosensation in Drosophila. Proc Natl Acad Sci U S A 93, 6079-6084.

Schepers, R.J., and Ringkamp, M. (2010). Thermoreceptors and thermosensitive afferents. Neuroscience and biobehavioral reviews 34, 177-184.

Takazawa, T., and MacDermott, A.B. (2010). Synaptic pathways and inhibitory gates in the spinal cord dorsal horn. Ann N Y Acad Sci 1198, 153-158.

Takemura, S.Y., Karuppudurai, T., Ting, C.Y., Lu, Z., Lee, C.H., and Meinertzhagen, I.A. (2011). Cholinergic circuits integrate neighboring visual signals in a Drosophila motion detection pathway. Curr Biol 21, 2077-2084.

Tang, X., Platt, M.D., Lagnese, C.M., Leslie, J.R., and Hamada, F.N. (2013). Temperature integration at the AC thermosensory neurons in Drosophila. J Neurosci 33, 894-901.

Thorne, N., and Amrein, H. (2008). Atypical expression of Drosophila gustatory receptor genes in sensory and central neurons. J Comp Neurol 506, 548-568.

Tian, L., Hires, S.A., Mao, T., Huber, D., Chiappe, M.E., Chalasani, S.H., Petreanu, L., Akerboom, J., McKinney, S.A., Schreiter, E.R., et al. (2009). Imaging neural activity in worms, flies and mice with improved GCaMP calcium indicators. Nat Methods 6, 875-881. 
Tichy, H. (2007). Humidity-dependent cold cells on the antenna of the stick insect. Journal of neurophysiology 97, 3851-3858.

Tichy, H., and Gingl, E. (2001). Problems in hygro- and thermoreception. In Ecology of sensing, F.G. Barth, and S.A. Berlin, eds. (Springer-Verlag), pp. 271-287.

Todd, A.J. (2010). Neuronal circuitry for pain processing in the dorsal horn. Nature reviews Neuroscience 11, 823-836.

van Vreeswijk, C., and Sompolinsky, H. (1996). Chaos in neuronal networks with balanced excitatory and inhibitory activity. Science 274, 1724-1726.

Venken, K.J., Simpson, J.H., and Bellen, H.J. (2011). Genetic manipulation of genes and cells in the nervous system of the fruit fly. Neuron 72, 202-230.

Werblin, F.S. (2010). Six different roles for crossover inhibition in the retina: correcting the nonlinearities of synaptic transmission. Visual neuroscience 27, 1-8.

Wilson, R.I., and Laurent, G. (2005). Role of GABAergic inhibition in shaping odor-evoked spatiotemporal patterns in the Drosophila antennal lobe. J Neurosci 25, 9069-9079.

Wilson, R.I., Turner, G.C., and Laurent, G. (2004). Transformation of olfactory representations in the Drosophila antennal lobe. Science 303, 366-370.

Xiang, Y., Yuan, Q., Vogt, N., Looger, L.L., Jan, L.Y., and Jan, Y.N. (2010). Light-avoidancemediating photoreceptors tile the Drosophila larval body wall. Nature 468, 921-926.

Yaksh, T.L. (1989). Behavioral and autonomic correlates of the tactile evoked allodynia produced by spinal glycine inhibition: effects of modulatory receptor systems and excitatory amino acid antagonists. Pain 37, 111-123.

Yasaka, T., Tiong, S.Y., Hughes, D.I., Riddell, J.S., and Todd, A.J. (2010). Populations of inhibitory and excitatory interneurons in lamina II of the adult rat spinal dorsal horn revealed by a combined electrophysiological and anatomical approach. Pain 151, 475-488.

Zaghloul, K.A., Boahen, K., and Demb, J.B. (2003). Different circuits for ON and OFF retinal ganglion cells cause different contrast sensitivities. The Journal of neuroscience : the official journal of the Society for Neuroscience 23, 2645-2654. 
Zars, T. (2001). Two thermosensors in Drosophila have different behavioral functions. J Comp Physiol A 187, 235-242. 


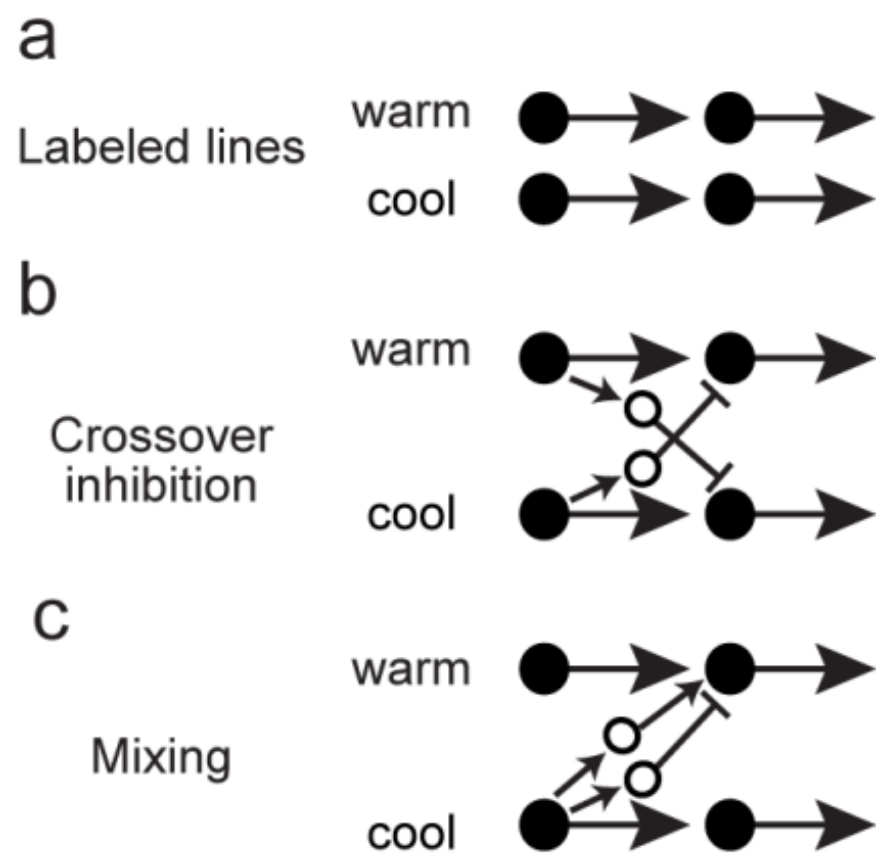

Figure 1: Putative computations implemented by thermosensory circuits. (Adapted from Prescott and Ratte, 2012)

(a) Warm and cool pathways are labeled lines do not interact.

(b) Warm-activated and cool-activated interneurons mediate crossover inhibition between warm and cool pathways.

(c) Excitatory and inhibitory interneurons mediate mixing between warm and cool inputs. 


\section{Figure 2: Jet micro-thermode schematic.}

a) Schematic showing the major components of the jet micro-thermode. A pressurized reservoir forces saline through the inner chamber of a heat exchanger. The saline is then expelled as a jet through a $160 \mu \mathrm{m}$ nozzle. This jet can be quickly turned on and off via a two-way solenoid valve (the "fast" valve). The outer chamber of the heat exchanger is fed by two pumps which circulate water from a hot and a cold water bath. Two three-way solenoid valves control the flow of water into the exchanger. A third valve (not shown) serves to return the outflow of the heat exchanger to the same bath that is feeding it.

b, c) In Hot Mode and Cold Mode the heat exchanger is heated or cooled by the continual circulation of water from one of the two baths through the outer chamber.

d) In the No Flow configuration, circulation in the outer chamber is stopped. This keeps the heat exchanger at a fairly constant temperature, stabilized by the thermal mass of the water in the outer chamber. 
a

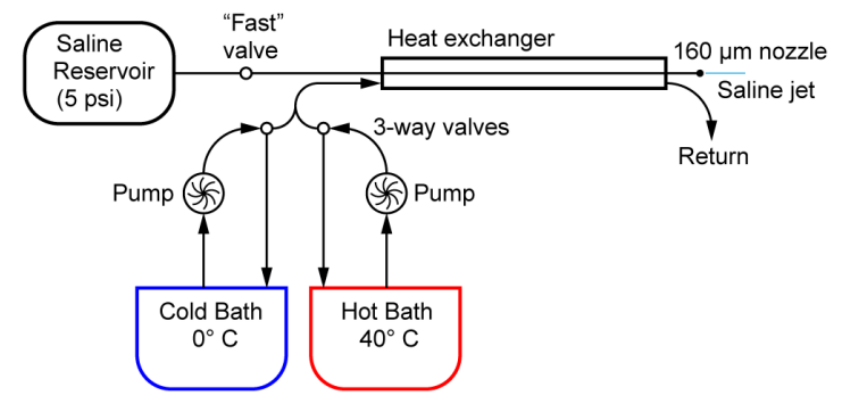

Jet micro-thermode

c

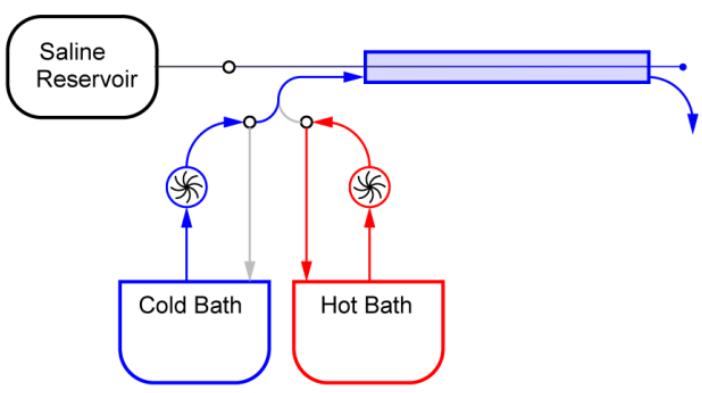

Cold Mode b

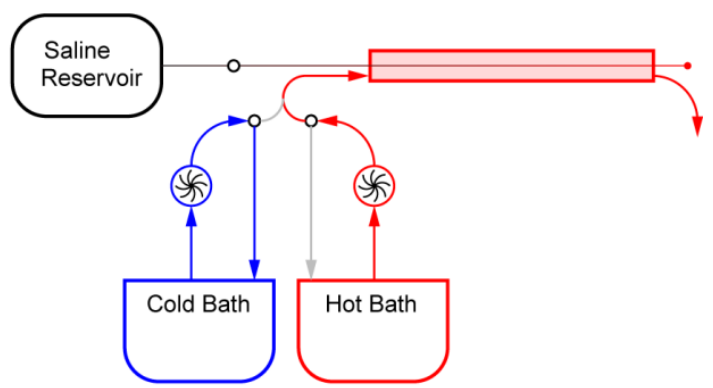

Hot Mode

d

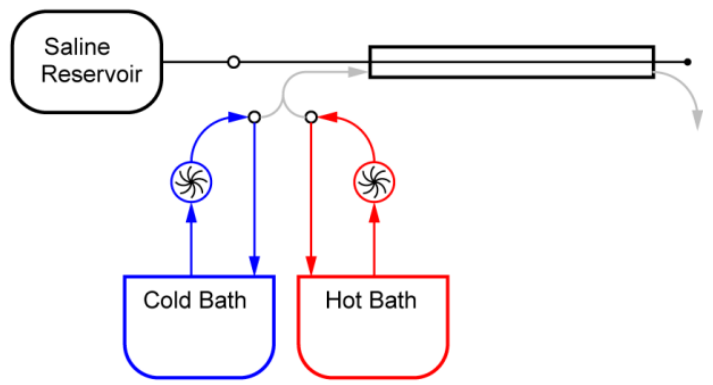

No Flow Mode

Figure 2: Jet micro-thermode schematic (continued). 
Figure 3: Morphologies of neurons in the proximal antennal protocerebrum.

Images are z-projections of coronal sections (dorsal is up). The soma remains sealed to the patch pipette at the end of each recording, and so the soma and the proximal part of the primary neurite are not present in the reconstructed morphologies; they are indicated schematically (soma with a black ball and primary neurite with a thick black line). Morphologies shown here were representative of all filled examples of the same neuron type (except as noted below). Regions are color-coded according to the key in (f).

a) A fast-cool-PN. These neurons have dendrites in the proximal antennal protocerebrum and axons projecting to two regions of the lateral protocerebrum (posterior lateral protocerebrum and posterior slope). Some fast-cool-PNs arborized unilaterally (rather than bilaterally) in the proximal antennal protocerebrum; we pooled data from both morphologies because their physiological properties were indistinguishable.

b) A slow-cool-PN. These neurons have dendrites in the ipsilateral proximal antennal protocerebrum and axons projecting to a subregion of the calyx of the mushroom body. This morphology has been described previously (Jenett et al., 2012) (that study called this neuron a "transverse antennal lobe projection neuron").

c) A warm-PN. These neurons have dendrites in the ipsilateral proximal antennal protocerebrum and axons projecting to two regions of the lateral protocerebrum (posterior lateral protocerebrum and posterior slope).

d) A warm-cool-PN. These neurons have dendrites in both the ipsi- and contralateral proximal antennal protocerebrum, as well as the ventral-posterior margin of the antennal lobe. Their axons project to two regions of the lateral protocerebrum (posterior lateral protocerebrum and posterior slope).

e) Two GABAergic LNs. All LNs we encountered in this study were bilateral. We define an "LN" here as a neuron that does not extend processes into any region except the proximal antennal protocerebrum (and the midline commissure that links them). Most LNs arborized broadly within the posterior antennal protocerebrum and so are well-positioned to mediate cross-talk between warm and cool pathways.

f) Locations of color-coded brain regions in a coronal section through the brain. 


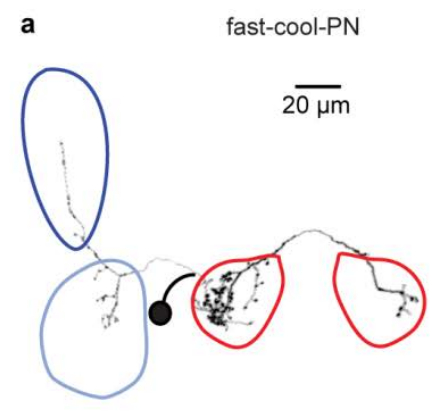

b
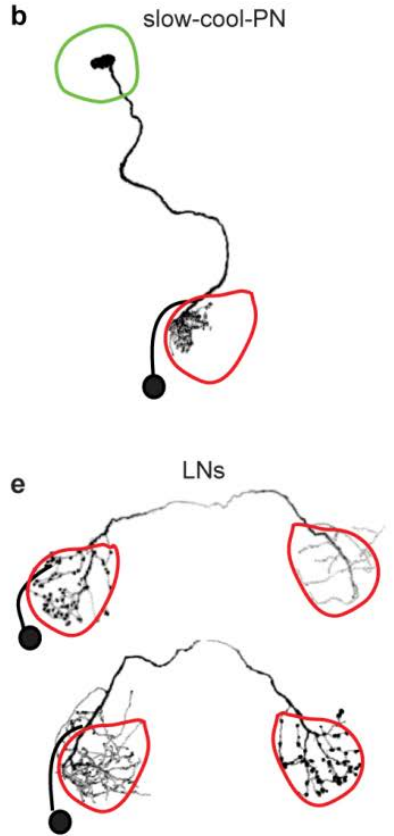

c warm-PN

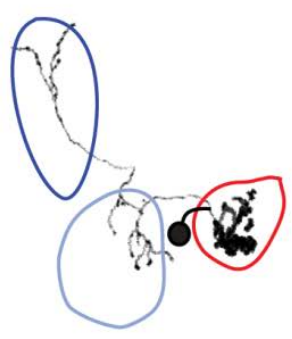

f

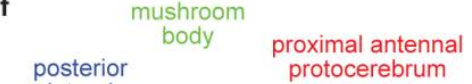

$$
\text { lateral }
$$

lateral
protocerebrum

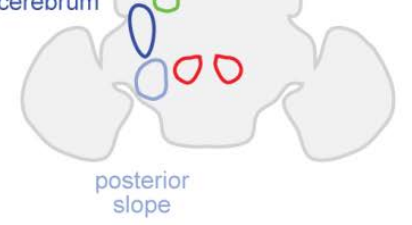

Figure 3: Morphologies of neurons in the proximal antennal protocerebrum (continued). 
Figure 4: Projection neurons excited by cooling.

(a) Fast-cool-PNs.

$\left(\mathrm{a}_{1}\right)$ In vivo recording from a fast-cool-PN. Stimuli are steps from $\sim 24^{\circ} \mathrm{C}$ to $\sim 13^{\circ} \mathrm{C}$ or $\sim 34^{\circ} \mathrm{C}$.

$\left(\mathrm{a}_{2}\right)$ Mean firing rate for the fast-cool-PNs, \pm s.e.m. across experiments $(n=7-10$ PNs per stimulus).

$\left(a_{3}\right)$ Sensitivity to small temperature steps: mean change in firing rate versus change in temperature, \pm s.e.m. $(n=5)$.

$\left(a_{4}\right)$ Proposed circuit for fast-cool-PNs (see Discussion regarding circuit diagrams).

(b) Slow-cool-PNs. Prolonged depolarization can elicit complex spikes $\left(b_{1}\right.$, inset, expanded 12fold). $\left(\mathrm{b}_{2}\right) n=7-14 ;\left(\mathrm{b}_{3}\right) n=7$. In ( $\left.\mathrm{b}_{4}\right)$, the smaller arrow represents a weaker connection. 

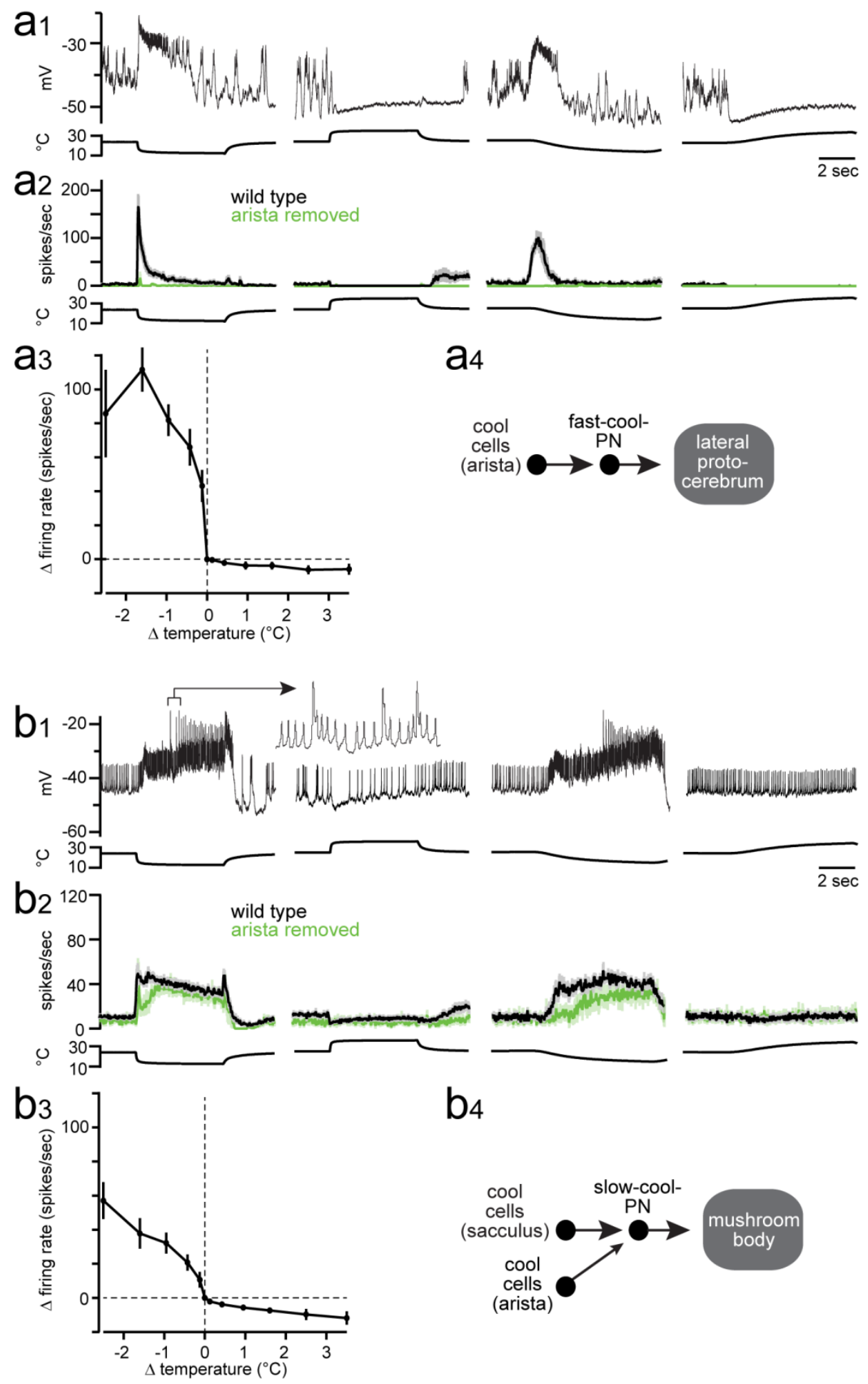

$\mathrm{b}_{4}$

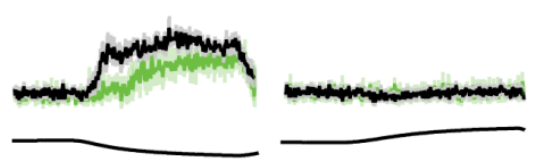

Figure 4: Projection neurons excited by cooling (continued). 

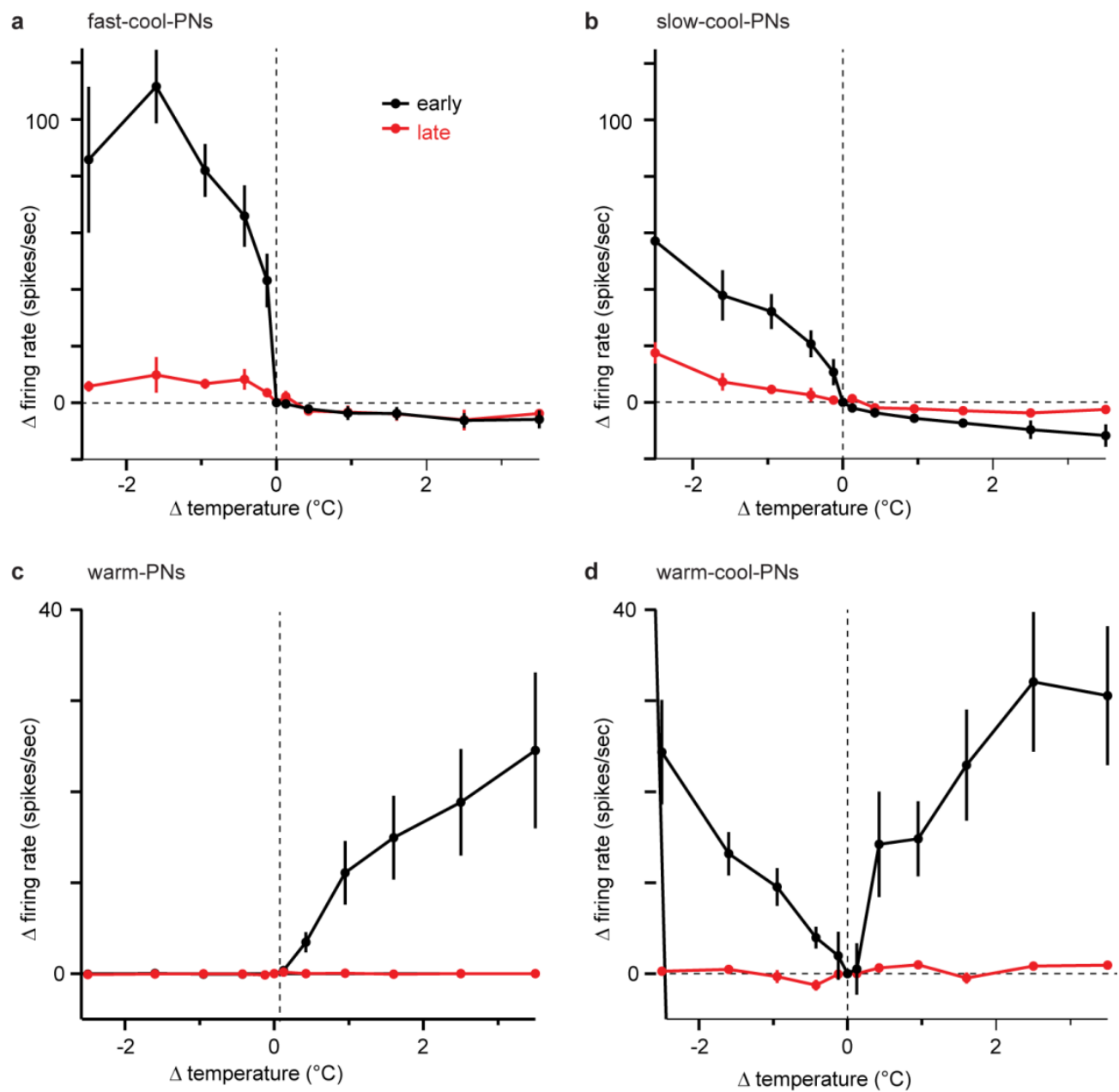

Figure 5: Adaptation to small temperature steps.

Sensitivity to small temperature steps, shown as the mean change in firing rate versus the change in temperature, averaged across experiments, \pm s.e.m. $(n=5-9)$. Data in red are measured during a late period after stimulus onset (from 4 to $5 \mathrm{~s}$ after valve opening). Data in black are measured during an early period after stimulus onset (from 100 to $400 \mathrm{~ms}$ after valve opening, except for the warm-cool-PNs, where it was from 100 to $200 \mathrm{~ms}$ ). Data in black are reproduced from Figure 4a (fast-cool-PNs), Figure 4b (slow-cool-PNs), Figure 8 (warm-PNs), and Figure 10 (warm-coolPNs). Note that slow-cool-PNs show little adaptation to large temperature steps (Figure $4 \mathrm{~b}$ ). However, their responses to small steps adapt strongly, like those of the other PN types. 
Figure 6: Innervation patterns in the proximal antennal protocerebrum.

Images are single coronal confocal sections through the proximal antennal protocerebrum, except where noted below. Magenta is neuropil (nc82 immunofluorescence). The approximate boundary of the proximal antennal protocerebrum is indicated in white outline.

a) Axon terminals of cold peripheral neurons and warm peripheral neurons. GFP expression is driven by specific Gal4 lines (GMR79C04-Gal4 for the cool cells, Gr28b.d-Gal4 for the warm cells). As noted previously (Gallio et al., 2011), the cool peripheral cells project to the lateral part of this brain region, whereas the warm peripheral cells project more medially. Neuropils surrounding the proximal antennal protocerebrum are labeled as LP (lateral protocerebrum), LAL (lateral accessory lobe) and SOG (subesophageal ganglion). Schematic at right shows a coronal section of the whole brain with the location of the proximal antennal protocerebrum indicated by white outlines.

b) Dendrites of three types of projection neurons. Each cell was filled with biocytin and visualized using a fluorescent streptavidin conjugate. The dendrites of the fast-cool-PNs reside in the lateral part of the proximal antennal protocerebrum. The same is true of the dendrites of the slow-cool-PNs (data not shown). The dendrites of the warm-PNs overlap with the axons of both warm and cool peripheral neurons. The dendrites of warm-cool-PNs are sparser than those of other PN types, so in a single section only scattered fragments of dendrite are visible; therefore a z-projection through the entire proximal antennal protocerebrum is shown in the image below. 
a axons of peripheral neurons
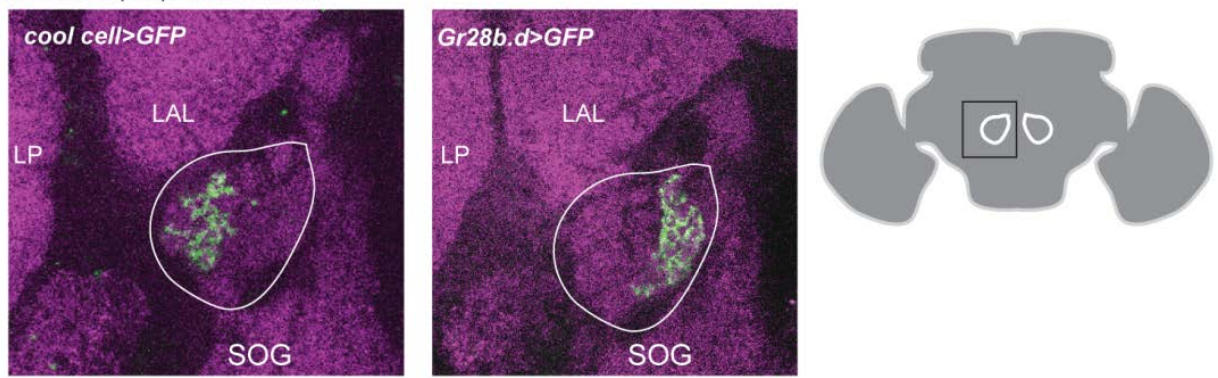

b dendrites of central neurons
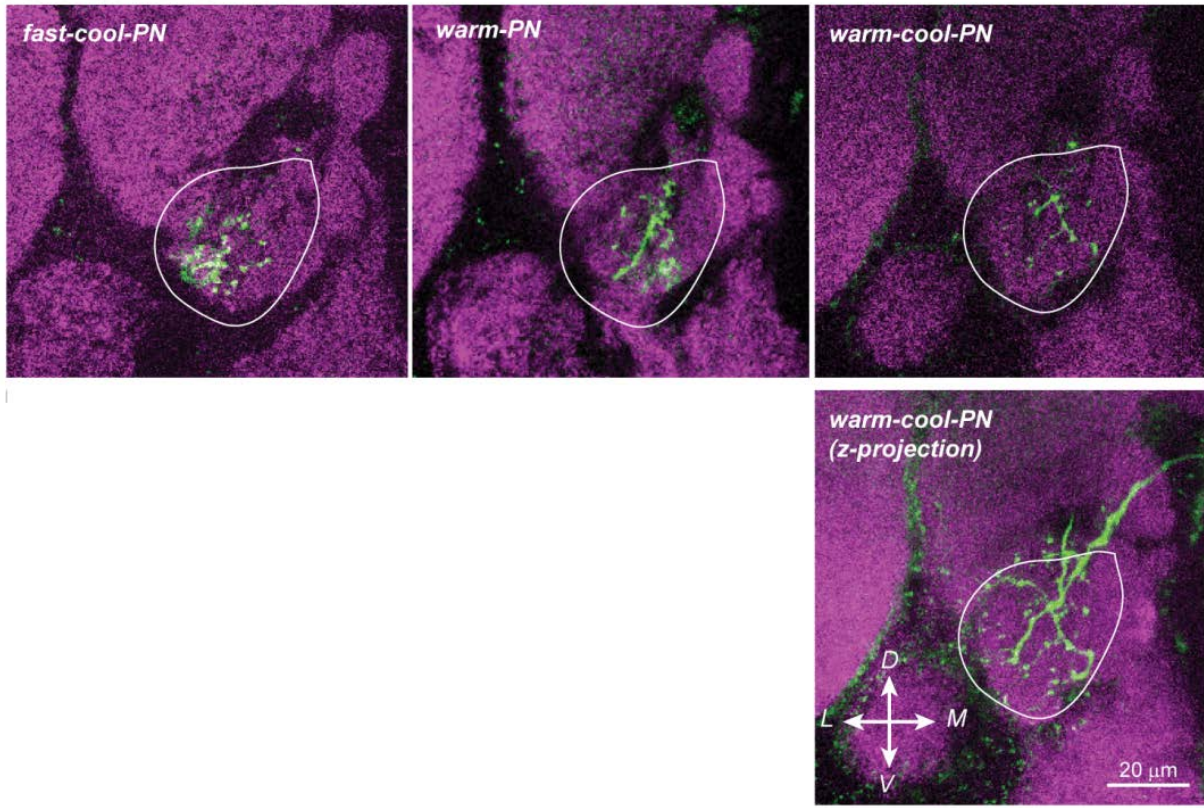

Figure 6: Innervation patterns in the proximal antennal protocerebrum (continued). 

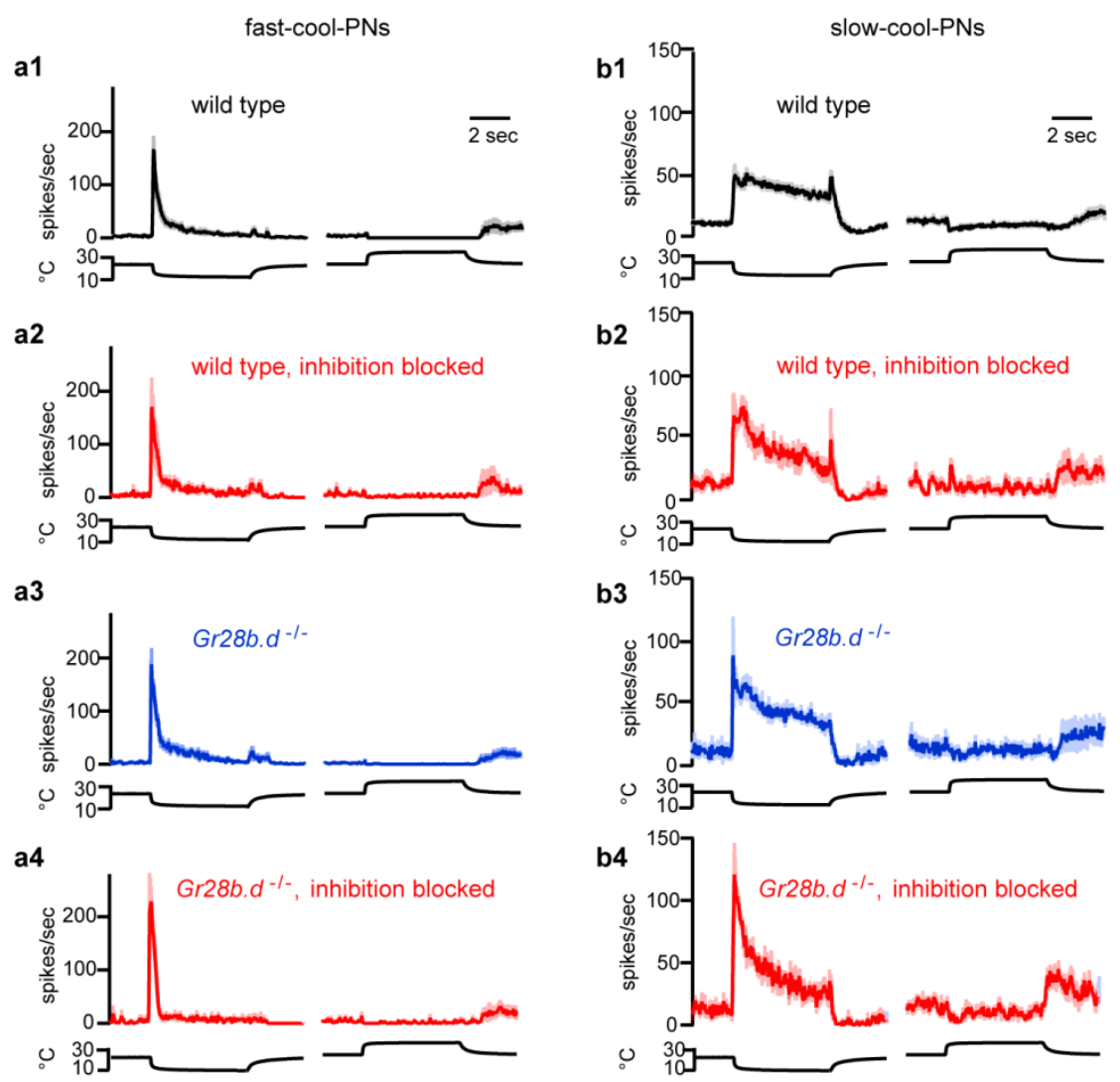

Figure 7: Circuit contributions to cool-PN responses.

(a) Fast-cool-PNs.

$\left(a_{1}\right)$ Mean firing rate for the fast-cool-PNs, \pm s.e.m. Reproduced from Figure 4a.

$\left(a_{2}\right)$ Mean firing rate with synaptic inhibition blocked with picrotoxin and CGP54626 $(n=4-$ $5)$.

$\left(\mathrm{a}_{3}\right)$ Mean firing rate in the $G r 28 b . d$ mutant $(n=5-10)$.

$\left(\mathrm{a}_{4}\right)$ Mean firing rate in the Gr28b.d mutant with inhibition blocked ( $\left.n=4-6\right)$.

(b) Slow-cool-PNs.

$\left(b_{1}\right)$ Mean firing rate for the slow-cool-PNs, \pm s.e.m. Reproduced from Figure $4 \mathrm{~b}$.

$\left(b_{2}\right)$ Mean firing rate with synaptic inhibition blocked with picrotoxin and CGP54626 $(n=4-$ 5).

$\left(\mathrm{b}_{3}\right)$ Mean firing rate in the Gr28b.d mutant ( $\left.n=4-5\right)$.

$\left(\mathrm{a}_{4}\right)$ Mean firing rate in the $G r 28 b . d$ mutant with inhibition blocked $(n=4)$.

In the Gr28b.d mutant, note that blocking inhibition produces only modest disinhibition in the cool-PNs. This contrasts with our results in the warm-PNs and warm-cool-PNs, where blocking inhibition in the mutant abolished all excitation evoked by the preferred stimulus for these neurons (Figure 8 and Figure 11). 
Figure 8: Projection neurons excited specifically by warming.

(a) A warm-PN. Cooling evokes a barrage of excitatory postsynaptic potentials (inset, expanded 4-fold).

(b) Mean firing rate for warm-PNs $(n=6-8)$.

(c) Mean firing rate with inhibition blocked using picrotoxin (an antagonist of GABA-A and GluCl receptors) and CGP54626 (a GABA-B antagonist) $(n=3-4)$. Spiking at cooling onset suggests excitation from the cool pathway. Spiking at cooling offset likely arises from the warm pathway and is normally masked by slowly-decaying cooling-evoked inhibition.

(d) Mean firing rate in the Gr28b.d mutant ( $n=5-7)$. The response to slow warming was smaller than wild type, although this trend did not reach statistical significance $(p=0.20)$. Blocking inhibition eliminates the excitatory response to fast warming steps $(n=4)$.

(e) Mean firing rate in genetic background for histamine experiments, before injecting histamine $(n=7)$. A histamine-gated chloride channel is expressed in warm thermoreceptor cells.

(f) Injecting histamine to silence warm thermoreceptor cells significantly reduces responses to slow warming ( $n=7, p<0.05$, paired t-test). Blocking inhibition eliminates warming-evoked excitation and unmasks cooling-evoked excitation $(n=6)$. See Figure 10 for controls.

(g) Sensitivity to small temperature steps (wild type $n=9$; wild type with inhibition blocked $n=$ 6; Gr28b.d mutant $n=5$, Gr28b.d mutant with inhibition blocked $n=3$ ). In wild type, blocking inhibition had no significant effect (unpaired $t$-tests, with iterative Bonferroni corrections; see Methods). However, responses were significantly smaller in the mutant near the threshold of warming-evoked excitation $\left(+0.425^{\circ} \mathrm{C}, p=0.017\right)$.

(h) Proposed circuit for the warm-PNs. 

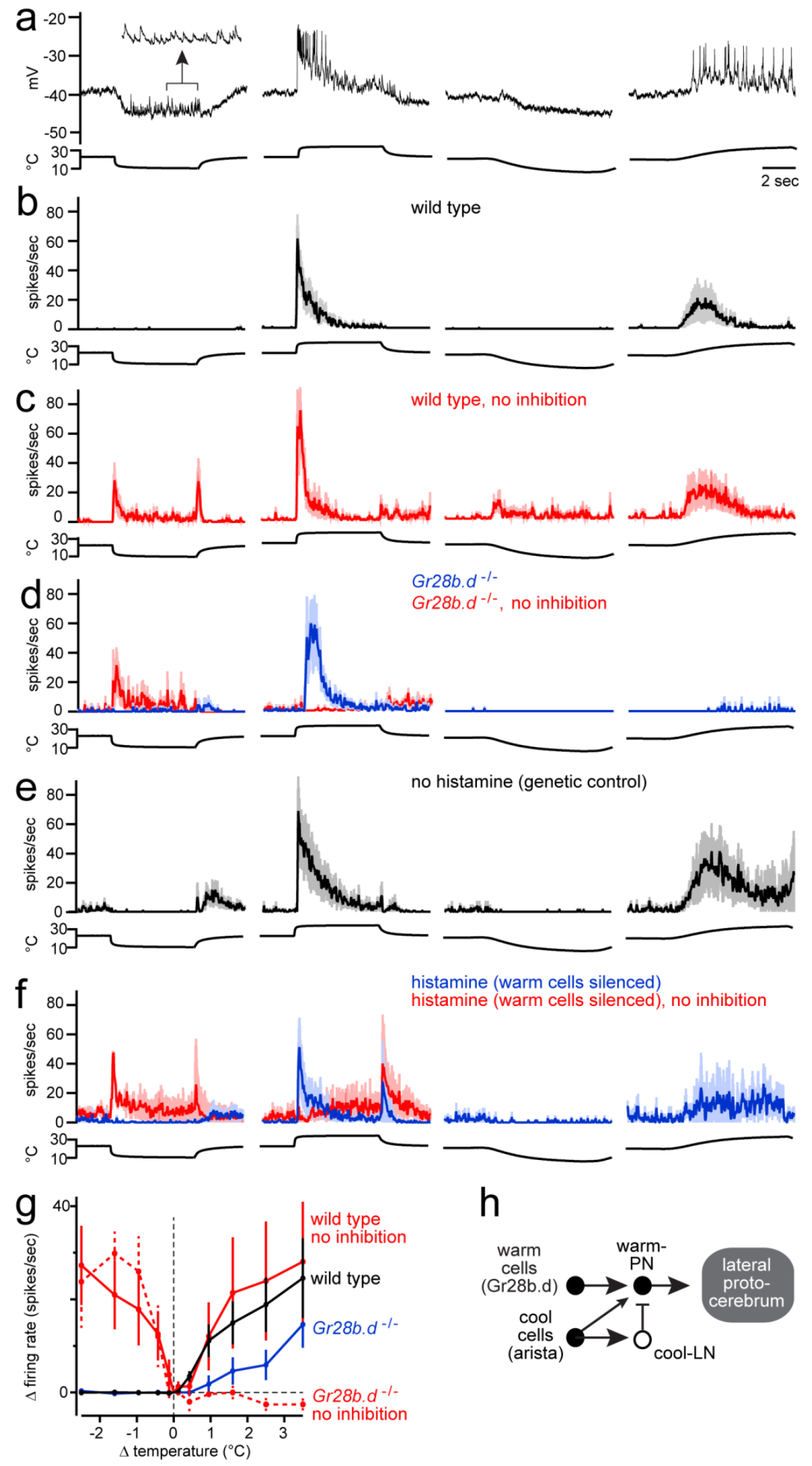

h

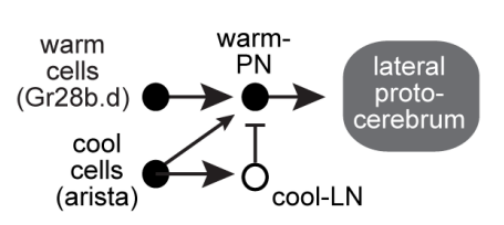

Figure 8: Projection neurons excited specifically by warming (continued). 

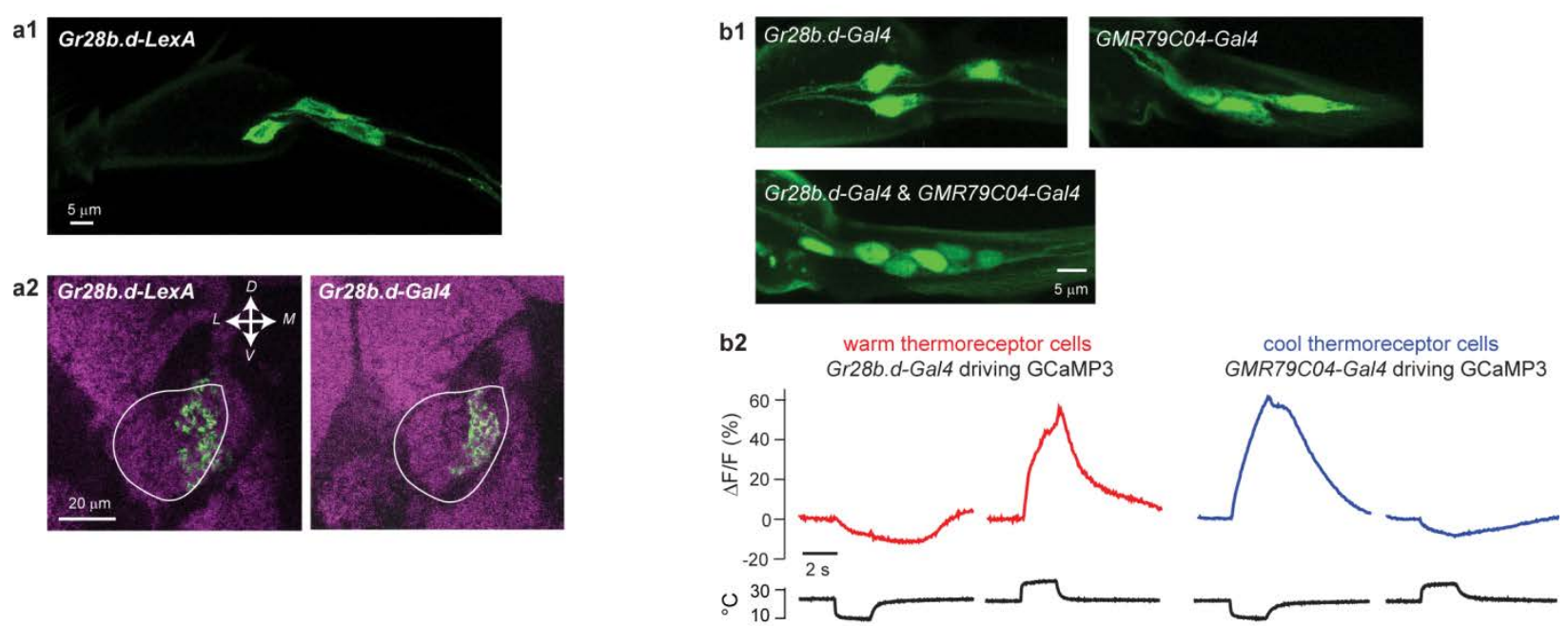

Figure 9: Validation of the LexA line for warm peripheral cells and the Gal4 line for cool peripheral cells.

There are 6 thermoreceptor cells at the base of the arista, comprising 3 warm cells and 3 cool cells. Gr28b.d-Gal4 labels the 3 warm cells.

(a) Validation of the Gr28b.d-LexA line for warm peripheral cells.

$\left(\mathrm{a}_{1}\right)$ We generated a LexA line using a Gr28b.d promoter fragment. Here we show that Gr28b.d-LexA labels 3 cells at the base of the arista. This image is a z-projection of a confocal stack through the base of the arista. Genotype is Gr28b.dLexA/+;26XLexAop2-mCD8::GFP/+.

$\left(\mathrm{a}_{2}\right)$ Axon terminals in the brain of peripheral neurons labeled by Gr28b.d-LexA (left) and Gr28b.d-Gal4 (right), both crossed with appropriate CD8::GFP reporter lines. As expected, the medial portion of the proximal antennal protocerebrum (outlined in white) is labeled with GFP. Images are single coronal confocal sections through the proximal antennal protocerebrum. Magenta is neuropil (nc82 immunofluorescence). Image on the right is reproduced from Figure 5.

(b) Validation of the GMR79C04-Gal4 line for cool peripheral cells.

(b) GMR79C04-Gal4 labels 3 cells at the base of the arista. Combining this driver with Gr28b.d-Gal4 labels 6 cells, indicating that these drivers label mutually exclusive populations of aristal neurons. These images show z-projections of confocal stacks through the base of the arista. Genotypes are Gr28b.d-Gal4/+;UAS-nls-GFP/+ (left), $U A S-n l s-G F P /+; G M R 79 C 04-G a l 4 /+$ (middle), Gr28b.d-Gal4/+;UAS-nlsGFP/GMR79C04-Gal4 (right).

$\left(\mathrm{b}_{2}\right)$ Calcium imaging of neurons at the base of the arista shows that Gr28b.d-Gal4 labels cells that are excited by warming and inhibited by cooling, whereas GMR79C04-Gal4 labels cells that are excited by cooling and inhibited by warming. Shown here are representative experiments using the "large fast step" stimuli. Genotypes are 20xUASGCaMP3/+;Gr28b.d-Gal4/+ (warm cell imaging) and 20XUASGCaMP3/+;;GMR79C04-Gal4/+ (cool cell imaging). Note that Figure 14g shows data for all experiments using "small fast step" stimuli. 
a
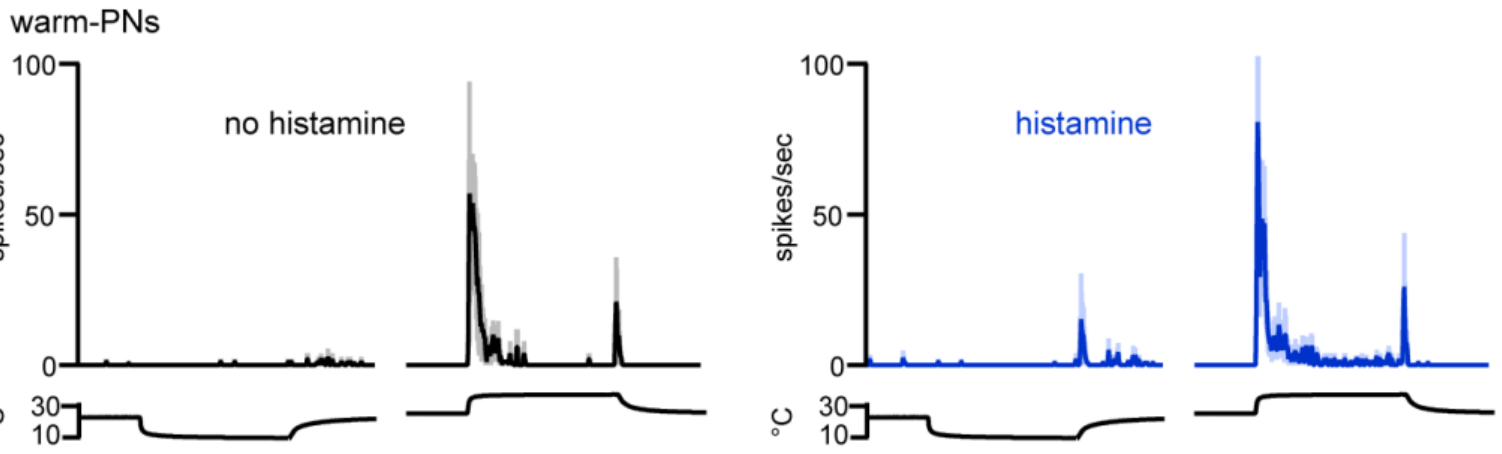

b warm-cool-PNs
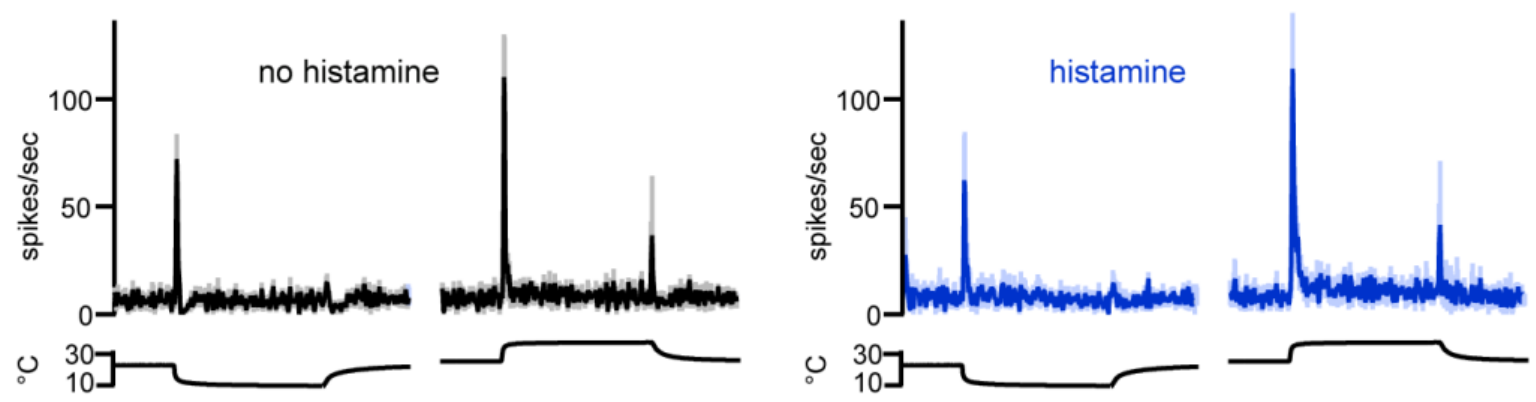

Figure 10: Histamine injection controls.

In these experiments the LexA driver (Gr28b.d-LexA) was omitted, but otherwise the experimental protocol was the same as in Figure 8e,f and Figure 11e-g. After histamine was injected into the antenna, there was little effect on the thermal responses of warm-PNs or warmcool-PNs.

a) Recordings from warm-PNs $(n=5)$.

Genotype is pLOT-HA-Ort/+;GMR95C02-Gal4,pJFRC2-10XUAS-IVS-mCD8::GFP.

b) Recordings from warm-cool-PNs $(n=4)$.

Genotype is pLOT-HA-Ort/+;GMR54A03-Gal4,pJFRC2-10XUAS-IVS-mCD8::GFP. 
Figure 11: Projection neurons excited by both warming and cooling.

(a) A warm-cool-PN. Fast warming and cooling elicit transient bursts of spikes (insets, expanded 7-fold). Stimulus offset does not elicit a response, probably because temperature is changing more slowly.

(b) Mean firing rate $(n=10-13)$.

(c) Mean firing rate with inhibition blocked $(n=3-5)$.

(d) Mean firing rate in the Gr28b.d mutant, before and after blocking inhibition ( $n=7-11$ and 4).

(e) Mean firing rate in genetic background for histamine experiments, before injecting histamine $(n=4)$. A histamine-gated chloride channel is expressed in warm thermoreceptor cells.

(f-g) Responses after injecting histamine to silence warm thermoreceptor cells $(n=4)$ and after blocking inhibition $(n=3)$. See Figure 10 for controls.

(h) Proposed circuit for the warm-cool-PNs. 

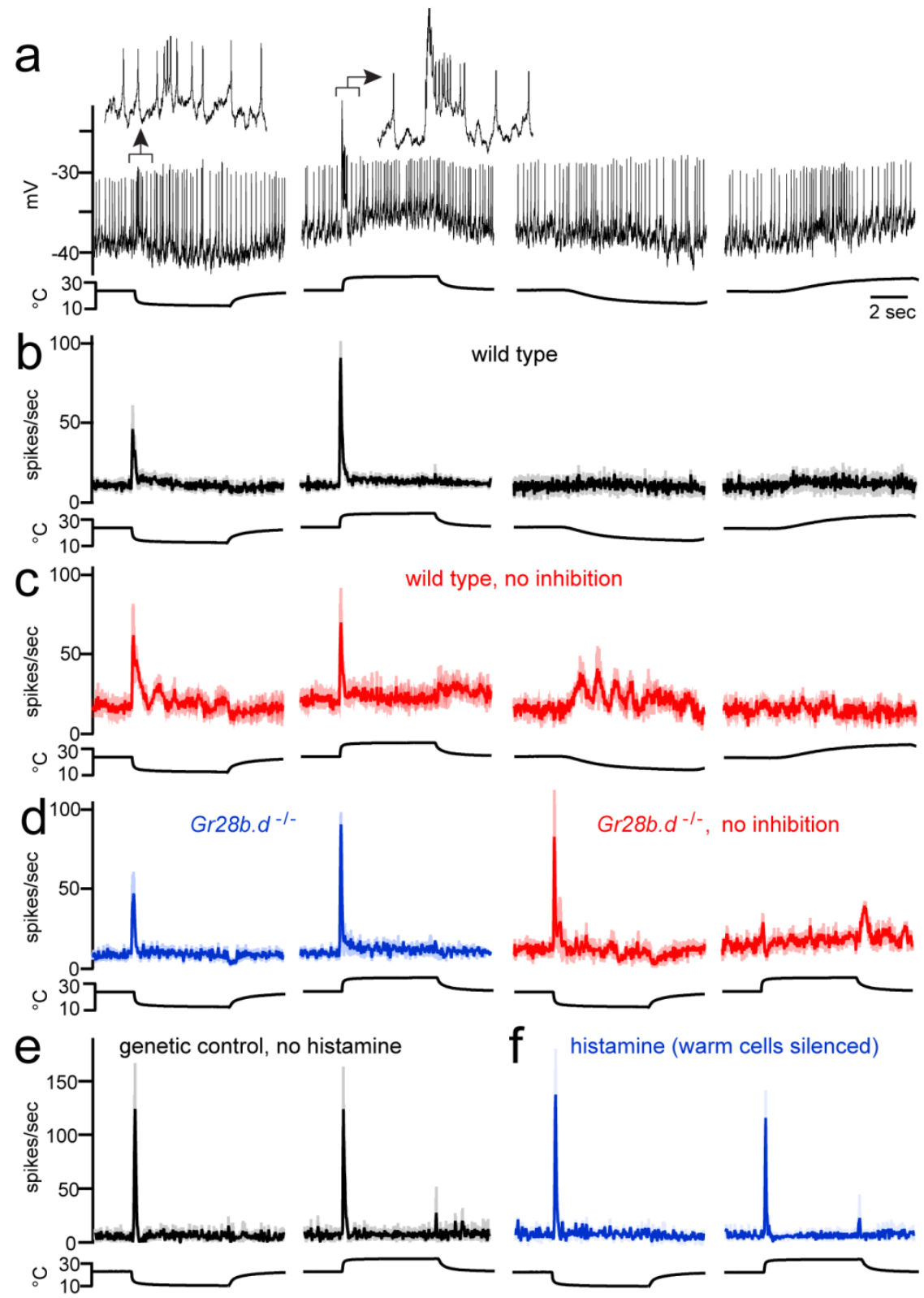

f histamine (warm cells silenced)
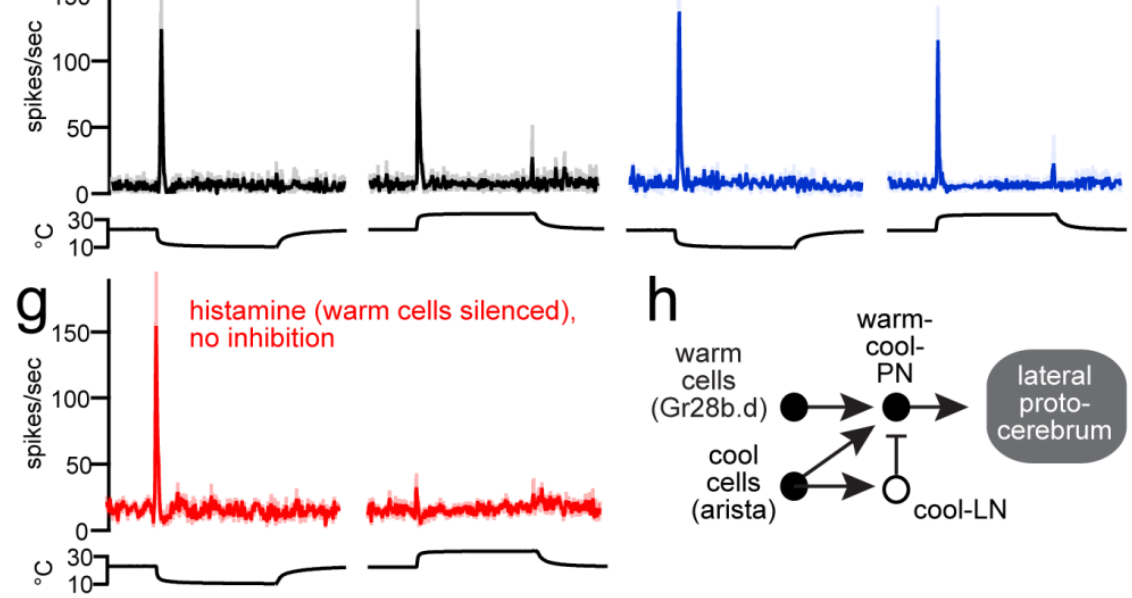

Figure 11: Projection neurons excited by both warming and cooling (continued). 


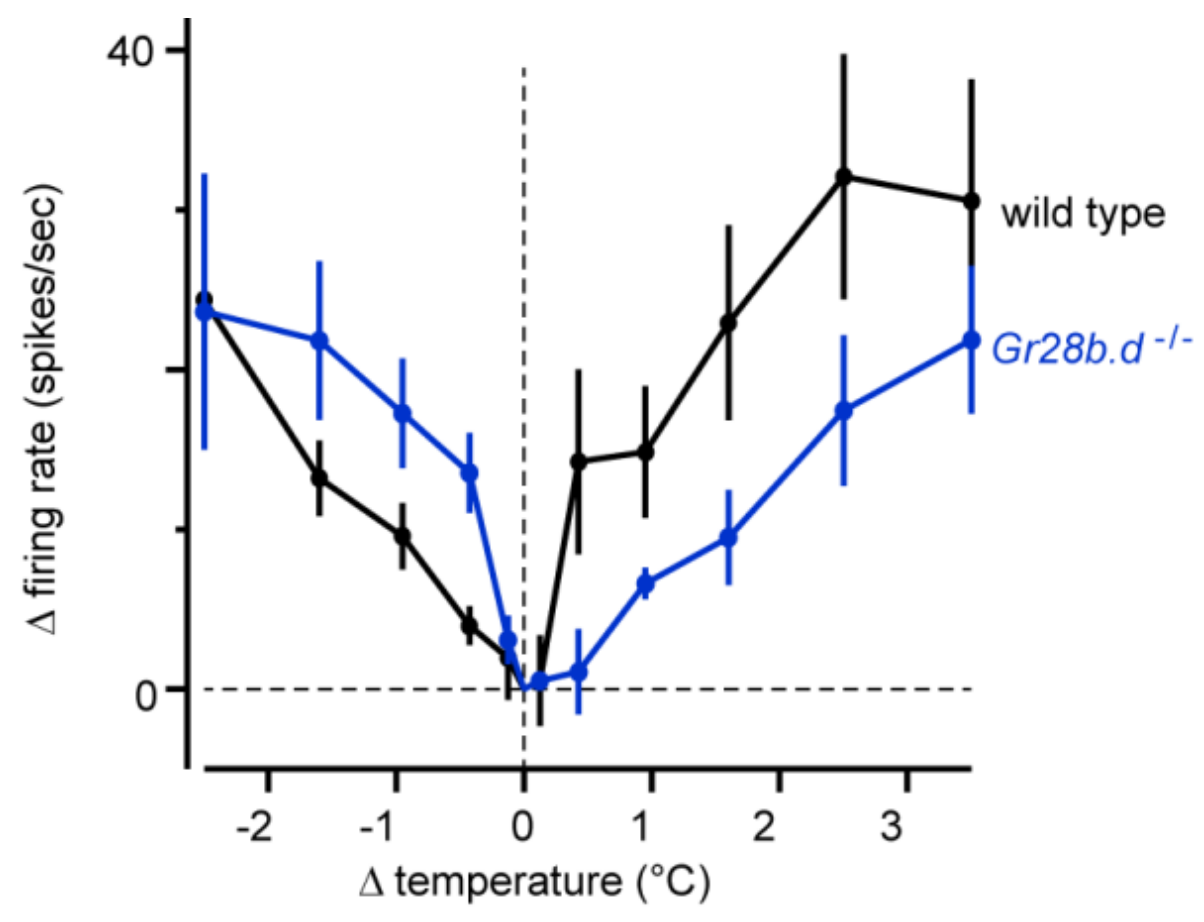

Figure 12: Sensitivity to small temperature steps in warm-cool-PNs.

Sensitivity to small temperature steps, shown as the mean change in firing rate versus the change in temperature, averaged across experiments, \pm s.e.m. $(n=5-9)$. Firing rate changes were averaged over a window from $100 \mathrm{~ms}$ to $200 \mathrm{~ms}$ after valve opening. Sensitivity was not significantly different in wild type versus the Gr28b.d mutant (unpaired $t$-tests with iterative Bonferroni corrections, see Methods). Blocking inhibition in these cells produced oscillatory activity that precluded analysis of responses to small steps with inhibition blocked. Genotypes are: GMR54A03-Gal4,pJFRC2-10XUAS-IVS-mCD8::GFP (wild type) and Gr28b ${ }_{\mathrm{MB} 03888}$;GMR54A03-Gal4,pJFRC2-10XUAS-IVS-mCD8::GFP (Gr28b.d $\left.d^{-/}\right)$ 
Gad1>CD8GFP

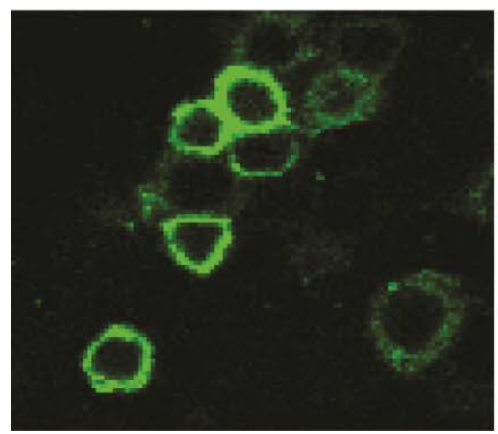

GABA

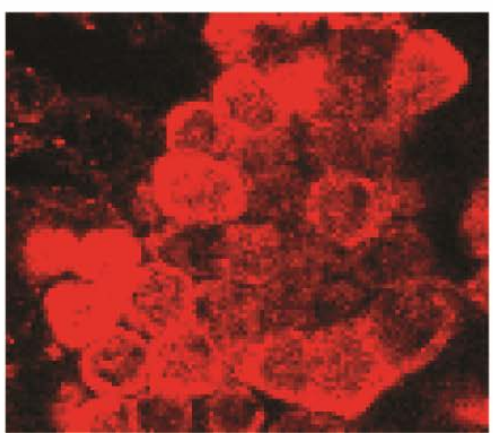

Merge

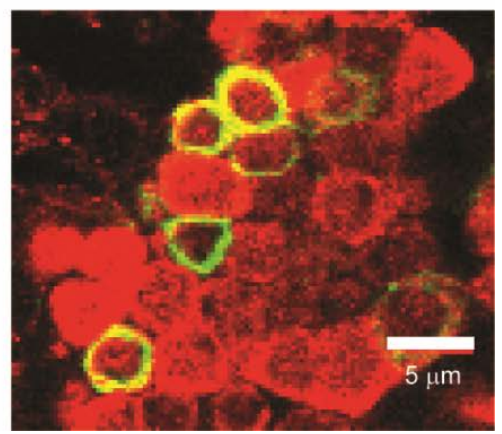

Figure 13: Validating the Gad1-Gal4 line as a marker of GABAergic local neurons in the posterior antennal protocerebrum.

In pilot studies, we found that all the local neurons we encountered in the posterior antennal protocerebrum had somata within a distinctive cluster ventral to the antennal lobes. We therefore targeted our electrodes to this cluster when we used a Gad1-Gal4 line to drive CD8::GFP expression in putative GABAergic neurons. In order to determine if the CD8::GFP-expressing somata in this cluster are indeed GABAergic, we performed dual immunofluorescence confocal microscopy with anti-CD8 and anti-GABA antibodies. This representative image shows that almost all CD8::GFP-expressing somata in this region are GABA-immunopositive (90\% overall), although not all GABAergic somata express CD8::GFP. This image is a single coronal section through the cluster of somata. 
Figure 14: Inhibitory local neurons responding to thermal stimuli.

(a) Recording from a GABAergic LN that is excited by cooling and inhibited by warming (a "cool-LN").

(b) Trial-averaged responses.

(c) Sensitivity to small temperature steps. Neurotransmitter release from these neurons may be related to both membrane potential and firing rate.

(d-f) Same as above, but for a different GABAergic LN.

(g) Sensitivity to small temperature steps in thermoreceptor cells of the arista, measured as the change in GCaMP3 fluorescence. See also Figure 9.

(h) Sensitivity to small temperature steps, measured from the membrane potential of fast-coolPNs, the more sensitive of the two cool-PN types. Each line represents a different experiment. The population of cool thermoreceptors must be at least this sensitive to warming. 


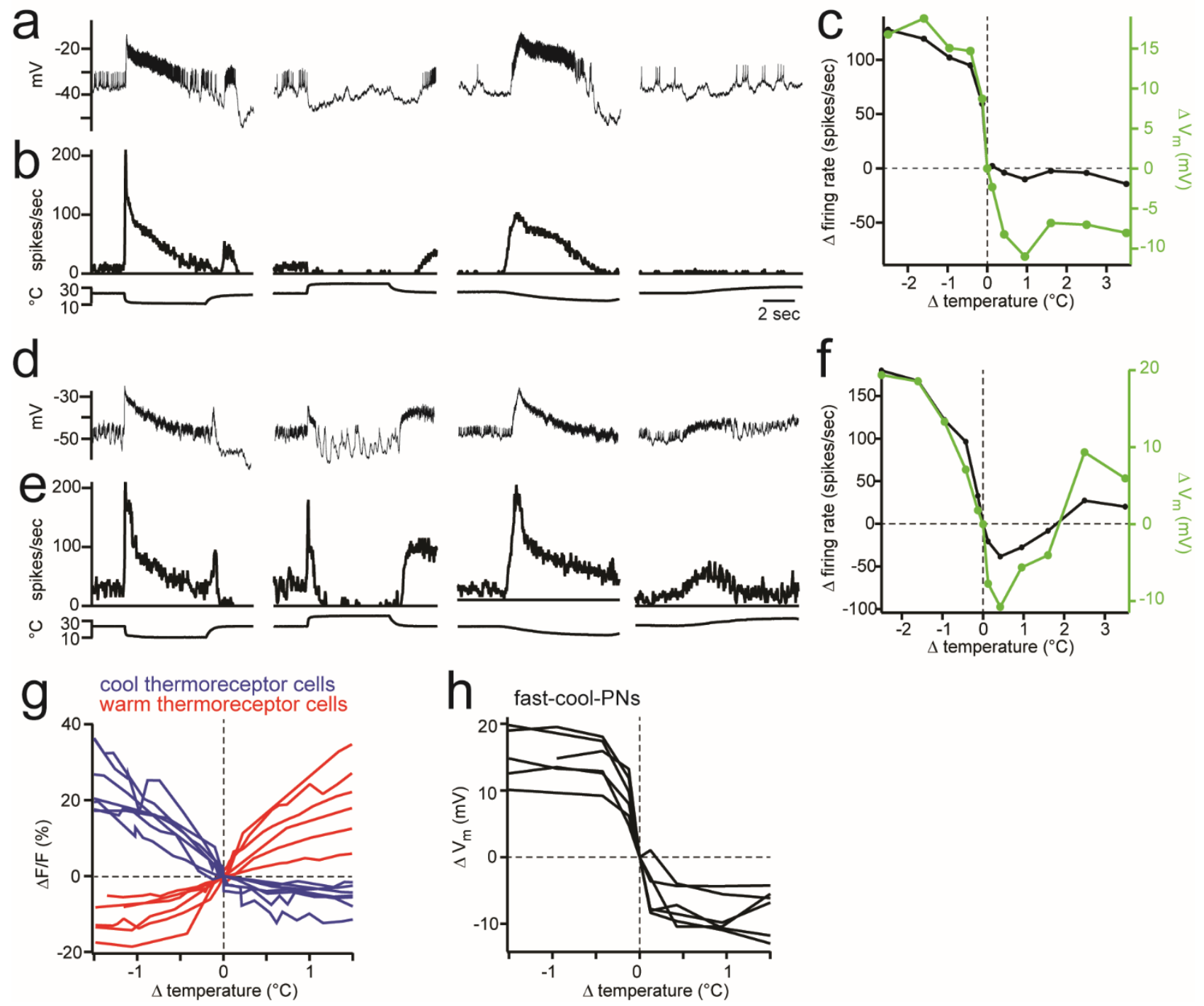

Figure 14: Inhibitory local neurons responding to thermal stimuli (continued). 

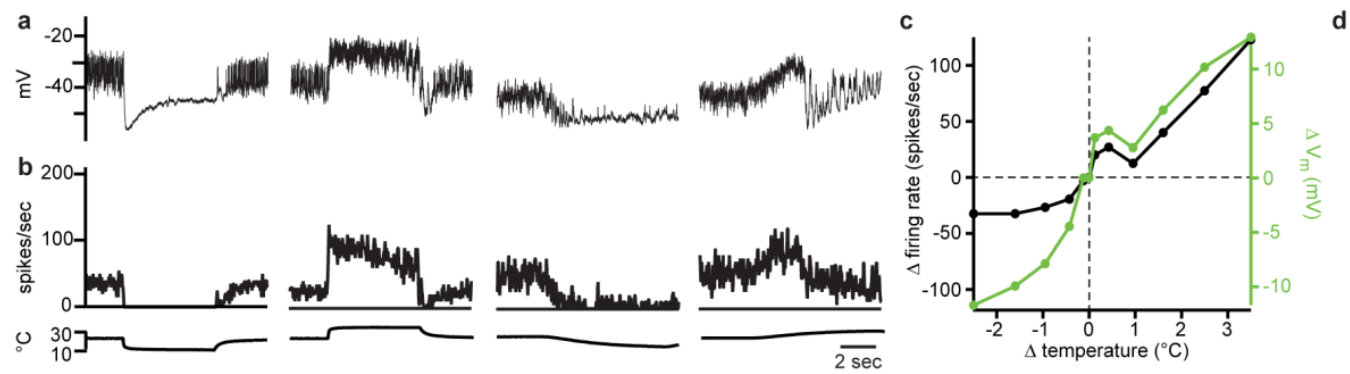

d
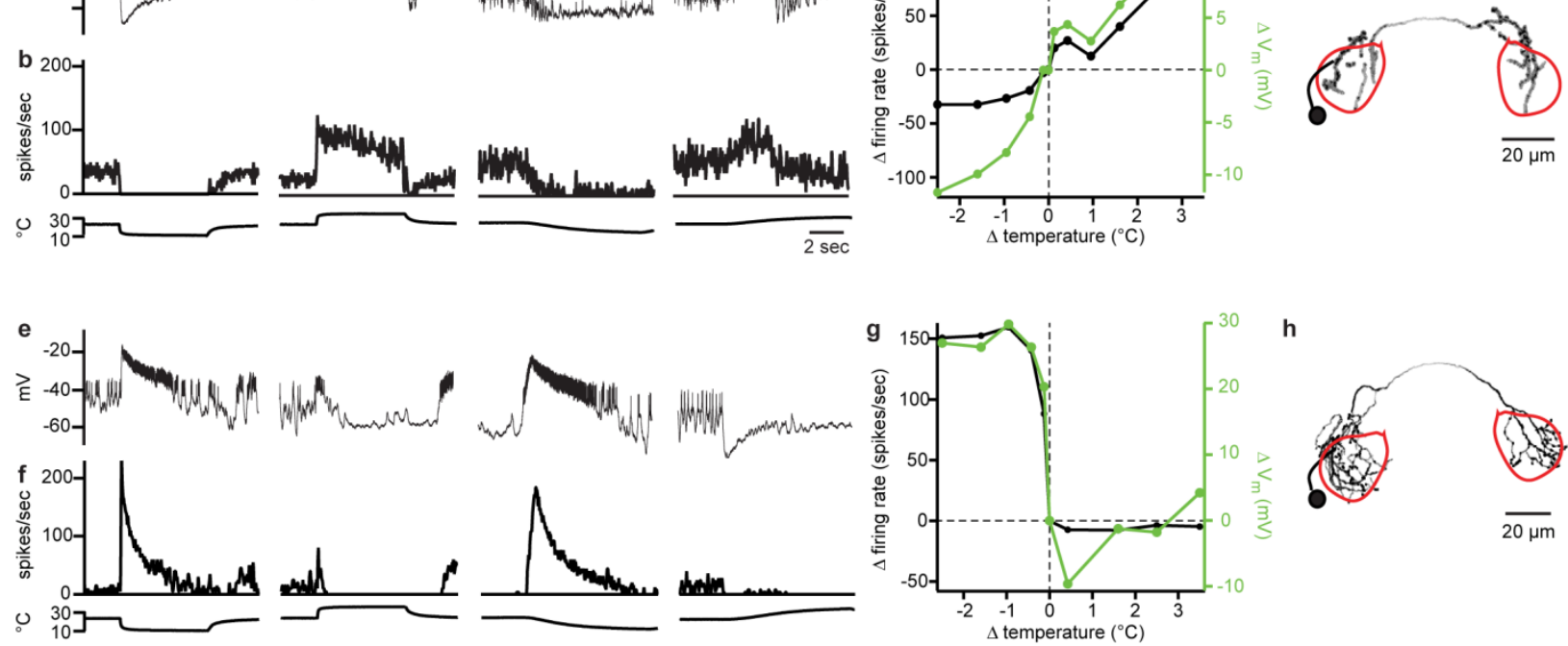

Figure 15: Additional examples of local neurons (LNs) in the proximal antennal protocerebrum.

For each LN, the sequence of panels is analogous to that of Figure 14: raw traces, peri-stimulus time histograms, and sensitivity plots. The morphologies of these cells ( $\mathrm{d}$ and $\mathrm{h}$ ) are displayed as in Figure 2, with a red outline indicating the boundary of the proximal antennal protocerebrum. a-d) A GABAergic LN. This LN is excited by warming and is inhibited by cooling. This cell was recorded in the genotype in $p J F R C 7-20 X U A S-I V S-m C D 8:: G F P /+$;Gadl-Gal4/+.

e-h) A glutamatergic LN. This LN is excited by cooling and is inhibited by warming, and its properties are similar to those of all the glutamatergic LNs we recorded from. In total we recorded from 6 glutamatergic LNs, three in the genotype GMR52G03-Gal4,pJFRC210XUAS-IVS-mCD8::GFP and three in the genotype GMR91H10-Gal4,pJFRC2-10XUASIVS-mCD8::GFP. 